\title{
NEW EXAMPLES OF MAXIMAL CURVES WITH LOW GENUS
}

\author{
DANIELE BARTOLI, MASSIMO GIULIETTI, MOTOKO KAWAKITA, AND MARIA MONTANUCCI
}

\begin{abstract}
Explicit equations for algebraic curves with genus 4, 5, and 10 that are either maximal or minimal over the finite field with $p^{2}$ elements are obtained for infinitely many $p$ 's. The key tool is the investigation of their Jacobian decomposition. Lists of small $p$ 's for which maximality holds are provided. In some cases we also describe the automorphism group of the curve.
\end{abstract}

\section{INTRODUCTION}

Throughout the paper, by curve we mean a projective, non-singular, geometrically irreducible algebraic curve defined over a finite field $\mathbb{F}_{q^{2}}$ of order $q^{2}$. A curve $\mathcal{X}$ with genus $g=g(\mathcal{X})$ is called $\mathbb{F}_{q^{2}}$-maximal if the number of its $\mathbb{F}_{q^{2}}$-rational points attains the Hasse-Weil upper bound, that is,

$$
\left|\mathcal{X}\left(\mathbb{F}_{q^{2}}\right)\right|=q^{2}+1+2 q g .
$$

Apart from being mathematical objects with intrinsic interest, maximal curves are often used for applications in Coding Theory, Cryptography, and Finite Geometry. For a survey on maximal curves we refer to [46, Chapter 10]. For a given $q$, the largest $g$ for which there exists an $\mathbb{F}_{q^{2}}$-maximal curve of genus $g$ is $q(q-1) / 2$ [49], and equality holds if and only if the curve is the Hermitian curve $\mathcal{H}_{q}$ with equation $X^{q+1}=Y^{q}+Y$ [69].

A complete solution of the classification problem for maximal curves seems to be out of reach and looking for new examples is still a very active line of research. A well-known construction method for maximal curves is based on a result of Kleiman [56], sometimes attributed to Serre (see [58]), stating that any non-singular curve which is $\mathbb{F}_{q^{2}}$-covered by an $\mathbb{F}_{q^{2}}$-maximal curve is also $\mathbb{F}_{q^{2}}$-maximal. Concrete examples of $\mathbb{F}_{q^{2}}$-maximal curves which are Galois-covered by $\mathcal{H}_{q}$ can be found e.g. in [14, 32, 59, 63]. For a long time the problem of establishing whether all $\mathbb{F}_{q^{2}}$-maximal curves were covered by $\mathcal{H}_{q}$ remained open; see e.g. [87]. The problem was solved in [35], where examples of $\mathbb{F}_{q^{2}}$-maximal curves, with $q=p^{3 h}>8, p$ a prime, non-covered by $\mathcal{H}_{q}$ were constructed. Applying Kleiman-Serre covering result to these curves and to their generalizations [30, [7] and [71] provided further examples of maximal curves; see [1,8, 15, 22, 38, 39]. Other recent constructions can be found in $42,78,81]$.

The aim of this paper is to provide new examples of $\mathbb{F}_{p^{2}}$-maximal curves with low genus. We remark

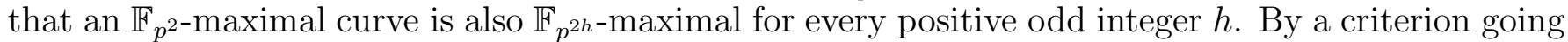
back to Tate [76. Theorem 2(d)] and explicitly pointed out by Lachaud [58, Proposition 5], a curve

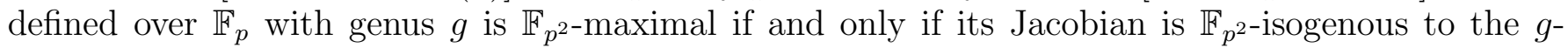
th power of an $\mathbb{F}_{p^{2}}$-maximal elliptic curve. Here, such a criterion is applied to plane curves whose

Date: December 10, 2019. 
Jacobian decomposition was originally studied in the zero characteristic case. In this direction, our main achievements are Theorem 2.4, Remark 2.5, Theorem 3.14, and Theorem 3.15. These results rely on a theorem by Kani and Rosen, which provides a decomposition of the Jacobian of a curve under some conditions on its automorphism group.

Theorem 1.1. [41, Theorem B] For a curve $\mathcal{X}$, let $G \leq A u t_{\mathbb{F}_{q}}(\mathcal{X})$ be a finite group such that $G=H_{1} \cup \cdots \cup H_{m}$, where the subgroups $H_{i}$ satisfy $H_{i} \cap H_{j}=\left\{1_{G}\right\}$ if $i \neq j$. Then we have the following $\mathbb{F}_{q}$-isogeny relation

$$
J_{\mathcal{X}}^{m-1} \times J_{\mathcal{X} / G}^{g} \simeq J_{\mathcal{X} / H_{1}}^{h_{1}} \times \cdots \times J_{\mathcal{X} / H_{m}}^{h_{m}}
$$

where $g=|G|, h_{i}=\left|H_{i}\right|$, and, as usual, $J^{r}=J \times \cdots J$ (r times).

Corollary 1.2. [41, Example 1] With the same notation as in Theorem 1.1, if $G$ is the direct product of two cyclic groups of order 2 , say $G=\left\{1_{G}, \alpha_{1}, \alpha_{2}, \alpha_{3}\right\}$, then

$$
J_{\mathcal{X}} \times J_{\mathcal{X} / G}^{2} \simeq J_{\mathcal{X} /\left\langle\alpha_{1}\right\rangle} \times J_{\mathcal{X} /\left\langle\alpha_{2}\right\rangle} \times J_{\mathcal{X} /\left\langle\alpha_{3}\right\rangle}
$$

Theorem 1.1 has already been used e.g. in [2, 10, 47, 48, 51, 54, 66] to describe examples of curves with many points with respect to their genus, including maximal curves. For some curves investigated in this work, Theorem 1.1 gives a decomposition of their Jacobian into elliptic curves. In order to establish whether these factors are isogeneous or not, we will use modular polynomials of low degrees, for which we refer to [12] and the web database [13].

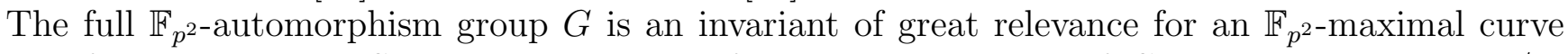
$\mathcal{X}$. In fact, by Kleiman-Serre covering result, for each subgroup $H$ of $G$ the quotient curve $\mathcal{X} / H$ is still $\mathbb{F}_{p^{2}}$-maximal. Therefore, automorphism groups of some of the curves under investigation are determined.

The paper is organized as follows. In Section 2 we study the curve $\mathcal{C}$ with equation

$$
\frac{4\left(x^{2}-x+1\right)^{3}}{27 x^{2}(x-1)^{2}}+\frac{4\left(y^{2}-y+1\right)^{3}}{27 y^{2}(y-1)^{2}}=1 \text {. }
$$

In characteristic zero, $\mathcal{C}$ is one of the two genus 4 curves with exactly 72 automorphisms and its Jacobian decomposes as the 4-th power of an elliptic curve; see [89]. We prove that such a curve is

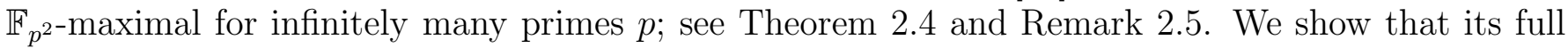
automorphism group is isomorphic to $\left(S_{3} \times S_{3}\right) \rtimes C_{2}$, as it happens in characteristic zero; see Section 2.2 .

In Section 3 we deal with a family of curves with genus 5, given by Equation (10). In characteristic zero, these curves were characterized by Shaska [70] as the genus 5 hyperelliptic curves with automorphism group isomorphic to the direct product of $A_{4}$ and a group of order 2; Paulhus later observed that their Jacobian decomposes in the product of three isogenous elliptic curves and a hyperelliptic curve of genus 2; see [67, Proposition 4]. The main result of the section is Theorem 3.14, which states that some curves in the family are either $\mathbb{F}_{p^{2}}$-maximal or $\mathbb{F}_{p^{2}}$-minimal (that is, attaining the Hasse-Weil lower bound) for infinitely many primes $p$. A list of $p$ for which specific curves of the family are $\mathbb{F}_{p^{2}}$-maximal is provided; see Theorem 3.15. In Section 4 we extend Shaska's characterization result to the positive characteristic case; see Theorem 4.2 . 
Finally, in Section 5 we report some results on the Jacobian decomposition and the $\mathbb{F}_{p^{2}}$-maximality of other curves with genus 10 .

\section{A CURVE OF GENUS 4}

In this section we study the plane curve $\mathcal{C}$ of the affine equation

$$
\frac{4\left(X^{2}-X+1\right)^{3}}{27 X^{2}(X-1)^{2}}+\frac{4\left(Y^{2}-Y+1\right)^{3}}{27 Y^{2}(Y-1)^{2}}=1
$$

over the finite fields $\mathbb{F}_{p}, p>7$ prime.

2.1. $\mathbb{F}_{p^{2}}$-maximality of $\mathcal{C}$. Let $F=\mathbb{F}_{p^{2}}(x, y)$ with $\left(4\left(x^{2}-x+1\right)^{3}\right) /\left(27 x^{2}(x-1)^{2}\right)+\left(4\left(y^{2}-y+\right.\right.$ $\left.1)^{3}\right) /\left(27 y^{2}(y-1)^{2}\right)=1$ be the function field of the curve $\mathcal{C}$. Let $\tau_{1}, \tau_{2}, \sigma$ be the order-2 automorphisms of $F$ defined by $\sigma(x, y)=(y, x), \tau_{1}(x, y)=(-x+1, y), \tau_{2}(x, y)=(x,-y+1)$. We consider the group $G=\left\langle\tau_{1}, \tau_{2}, \sigma\right\rangle$. Such a group is elementary abelian of order 8 and can be written as

$$
G=\left\langle\tau_{1}\right\rangle \cup\left\langle\tau_{2}\right\rangle \cup\langle\sigma\rangle \cup\left\langle\sigma \tau_{1}\right\rangle \cup\left\langle\sigma \tau_{1} \tau_{2}\right\rangle,
$$

where $\left\langle\sigma \tau_{1}\right\rangle=\left\{i d, \sigma \tau_{1}, \tau_{1} \tau_{2}, \sigma \tau_{2}\right\}$ and $\left|\left\langle\tau_{1}\right\rangle\right|=\left|\left\langle\tau_{2}\right\rangle\right|=|\langle\sigma\rangle|=\left|\left\langle\sigma \tau_{1} \tau_{2}\right\rangle\right|=2$.

Proposition 2.1. The Jacobian variety of both the quotient curves $\mathcal{C} /\left\langle\tau_{1}\right\rangle$ and $\mathcal{C} /\left\langle\tau_{2}\right\rangle$ is $\mathbb{F}_{p}$-isogenous to twice the Jacobian variety of the elliptic curve with equation

$$
Y^{2}=X^{3}+\frac{9}{16} X^{2}+\frac{3}{16} X+\frac{1}{64} .
$$

Proof. Note that $\tau_{1}$ fixes both $y_{1}=y$ and $x_{1}=x(-x+1)$. As $\tau_{1}$ is an involution, the function field of $\mathcal{C} / \tau_{1}$ over $\overline{\mathbb{F}}_{p}$ is $\mathbb{F}_{p}$-isomorphic to $\overline{\mathbb{F}}_{p}\left(x_{1}, y_{1}\right)$. A straightforward computation gives

$$
\left.4\left(\left(-y_{1}+1\right)^{3} x_{1}^{2}\left(x_{1}-1\right)^{2}+\left(x_{1}^{2}-x_{1}+1\right)^{3} y_{1}^{2}\right)-27 y_{1}^{2} x_{1}^{2}\left(x_{1}-1\right)^{2}\right)=0 .
$$

Consider the genus 2 hyperellittic function field $\overline{\mathbb{F}}_{p}(u, v)$ with

$$
v^{2}=4 u^{6}-12 u^{5}+21 u^{4}-22 u^{3}+21 u^{2}-12 u+4,
$$

and let $\mathcal{G}: Y^{2}=4 X^{6}-12 X^{5}+21 X^{4}-22 X^{3}+21 X^{2}-12 X+4$ be the corresponding hyperelliptic curve of genus 2 . Then $\overline{\mathbb{F}}_{p}\left(x_{1}, y_{1}\right) \cong \overline{\mathbb{F}}_{p}(u, v)$ via the $\mathbb{F}_{p}$-isomorphism $\psi: \overline{\mathbb{F}}_{p}(u, v) \rightarrow \overline{\mathbb{F}}_{p}(\xi, \eta)$ defined by

$$
\psi(u, v)=\left(\frac{a_{1}(u, v)}{a_{3}(u, v)}, \frac{a_{2}(u, v)}{a_{3}(u, v)}\right),
$$

with 


$$
\begin{aligned}
a_{1}(u, v)= & \frac{1}{2} u^{8}-\frac{3}{2} u^{7}+\frac{21}{8} u^{6}-\frac{1}{4} u^{5} v-\frac{13}{4} u^{5}+\frac{3}{8} u^{4} v+\frac{17}{4} u^{4} \\
& +\frac{1}{8} u^{3} v-\frac{13}{4} u^{3}+\frac{1}{8} u^{2} v+\frac{21}{8} u^{2}+\frac{3}{8} u v-\frac{3}{2} u-\frac{1}{4} v+\frac{1}{2}, \\
a_{2}(u, v)= & u^{6}-\frac{3}{2} u^{5}+u^{4}+\frac{1}{2} u^{3} v-\frac{1}{2} u^{3}-\frac{1}{2} u^{2} v, \\
a_{3}(u, v)= & u^{6}-2 u^{5}+2 u^{4}-2 u^{3}+u^{2} .
\end{aligned}
$$

Hence $\mathcal{G} \cong \mathcal{C} /\left\langle\tau_{1}\right\rangle$ and applying the same argument to $x_{2}=x, y_{2}=y(-y+1)$, and $\tau_{2}$, we see that $\mathcal{G} \cong \mathcal{C} /\left\langle\tau_{2}\right\rangle$ as well.

Now we investigate the Jacobian decomposition of the hyperellittic curve $\mathcal{G}$. We apply Corollary 1.2 to $\mathcal{G}$ and the automorphism group of order 4 generated by the involutions

$$
\psi_{1}(u, v)=(u,-v), \quad \psi_{2}(u, v)=(-u+1, v) .
$$

It is immediate to see that the quotient curve $\mathcal{G} /\left\langle\psi_{1}\right\rangle$ is rational, whereas both $\mathcal{G} /\left\langle\psi_{2}\right\rangle$ and $\mathcal{G} /\left\langle\psi_{1} \psi_{2}\right\rangle$ are elliptic. In fact, $\psi_{2}$ fixes $u_{1}=-u^{2}+u$ and $v_{1}=v$, and

$$
v_{1}^{2}=-4 u_{1}^{3}+9 u_{1}^{2}-12 u_{1}+4
$$

holds; also, $\psi_{1} \psi_{2}$ fixes $u_{2}=u_{1}$ and $v_{2}=v(2 u-1)$ and

$$
v_{2}^{2}=\left(-4 u_{2}^{3}+9 u_{2}^{2}-12 u_{2}+4\right)\left(-4 u_{2}+1\right) .
$$

The function fields of the curves $\mathcal{G} /\left\langle\psi_{2}\right\rangle$ and $\mathcal{G} /\left\langle\psi_{1} \psi_{2}\right\rangle$ are $\mathbb{F}_{p}$-isomorphic to $\overline{\mathbb{F}}_{p}\left(\mathcal{C}_{1}\right)=\overline{\mathbb{F}}_{p}\left(x^{\prime}, y^{\prime}\right)$ and $\overline{\mathbb{F}}_{p}\left(\mathcal{C}_{2}\right)=\overline{\mathbb{F}}_{p}\left(x^{\prime \prime}, y^{\prime \prime}\right)$ with

and

$$
\left(y^{\prime}\right)^{2}=\left(x^{\prime}\right)^{3}+\frac{9}{16}\left(x^{\prime}\right)^{2}+\frac{3}{16}\left(x^{\prime}\right)+\frac{1}{64}
$$

respectively, where

$$
\left(y^{\prime \prime}\right)^{2}=\left(x^{\prime \prime}\right)^{3}+\frac{11}{12}\left(x^{\prime \prime}\right)^{2}+\frac{1}{9}\left(x^{\prime \prime}\right)+\frac{1}{81},
$$

$$
x^{\prime}=-\frac{1}{4} u_{1}, \quad y^{\prime}=-\frac{1}{16} v_{1}, \quad x^{\prime \prime}=\frac{1 / 6 u_{2}-1 / 24}{-\left(u_{2}\right)^{2}+1 / 2 u_{2}-1 / 16}, \quad y^{\prime \prime}=\frac{1 / 36 v_{2}}{-\left(u_{2}\right)^{2}+1 / 2 u_{2}-1 / 16} .
$$

The two elliptic curves $\mathcal{C}_{1}$ and $\mathcal{C}_{2}$ are isogenous via the isogeny defined over their function field by $\theta\left(x^{\prime \prime}, y^{\prime \prime}\right)=\left(\theta_{1}\left(x^{\prime \prime}, y^{\prime \prime}\right), \theta_{2}\left(x^{\prime \prime}, y^{\prime \prime}\right)\right)$ where

$$
\begin{aligned}
& \theta_{1}=\frac{1 / 4\left(x^{\prime \prime}\right)^{3}-1 / 9 x^{\prime \prime}}{\left(x^{\prime \prime}+2 / 9\right)^{2}} \\
& \theta_{2}=\frac{1 / 8\left(x^{\prime \prime}\right)^{3} y^{\prime \prime}+1 / 12\left(x^{\prime \prime}\right)^{2} y^{\prime \prime}+1 / 18\left(x^{\prime \prime}\right) y^{\prime \prime}-1 / 81 y^{\prime \prime}}{\left(x^{\prime \prime}+2 / 9\right)^{3}}
\end{aligned}
$$

By Corollary 1.2, the claim follows. 
Proposition 2.2. The Jacobian varieties of both the quotient curves $\mathcal{C} /\langle\sigma\rangle$ and $\mathcal{C} /\left\langle\sigma \tau_{1} \tau_{2}\right\rangle$ are $\mathbb{F}_{p^{-}}$ isogenous to the Jacobian variety of the elliptic curve of equation

$$
Y^{2}=X^{3}+\frac{9}{16} X^{2}+\frac{3}{16} X+\frac{1}{64} .
$$

Proof. First note that the curves $\mathcal{C} /\langle\sigma\rangle$ and $\mathcal{C} /\left\langle\sigma \tau_{1} \tau_{2}\right\rangle$ are $\mathbb{F}_{p}$-isomorphic since the subgroups $\langle\sigma\rangle$ and $\left\langle\sigma \tau_{1} \tau_{2}\right\rangle$ are conjugated in the $\mathbb{F}_{p}$-automorphism group of $\mathcal{C}$. Indeed $\gamma \sigma=\tau_{1} \tau_{2} \gamma$, where $\gamma(x, y)=$ $(x,-y+1)$ is an automorphism of $F$. Therefore, we are going to deal only with the curve $\mathcal{C} /\langle\sigma\rangle$.

Both $x_{2}=x+y$ and $y_{2}=x y$ are fixed by $\sigma$. Then $\overline{\mathbb{F}}_{p}\left(x_{2}, y_{2}\right) \cong \overline{\mathbb{F}}_{p}(\mathcal{C} /\langle\sigma\rangle)$. Also,

$$
\begin{aligned}
& 4 x_{2}^{4} y_{2}^{2}+4 x_{2}^{4}-8 x_{2}^{3} y_{2}^{3}-12 x_{2}^{3} y_{2}^{2}-12 x_{2}^{3} y_{2}-8 x_{2}^{3}+4 x_{2}^{2} y_{2}^{4}+8 x_{2}^{2} y_{2}^{3}+21 x_{2}^{2} y_{2}^{2}+8 x_{2}^{2} y_{2} \\
& \quad+4 x_{2}^{2}+12 x_{2} y_{2}^{4}+14 x_{2} y_{2}^{3}+14 x_{2} y_{2}^{2}+12 x_{2} y_{2}-8 y_{2}^{5}-19 y_{2}^{4}-38 y_{2}^{3}-19 y_{2}^{2}-8 y_{2}=0 .
\end{aligned}
$$

As $\sigma$ fixes exactly 6 points of $\mathcal{C}$, by the Riemann-Hurwitz formula the genus of $\mathcal{C} /\langle\sigma\rangle$ is 1 .

An isogeny $\varphi$ between $\mathcal{C} /\langle\sigma\rangle$ (with homogeneous coordinates $X_{1}, Y_{1}$ and $Z_{1}$ ) and the elliptic curve

$$
Y^{2} Z=X^{3}+\frac{9}{16} X^{2} Z+\frac{3}{16} X Z^{2}+\frac{1}{64} Z^{3}
$$

can be constructed as follows. Consider the map $\varphi_{1}$ given by

$$
\begin{aligned}
& \varphi_{1}\left(X_{2}\right)=2^{5} \cdot 7^{4} X_{2}^{3} Y_{2}^{5} Z_{2}-2^{4} \cdot 3 \cdot 7^{4} X_{2}^{2} Y_{2}^{6} Z_{2}-2^{4} \cdot 7^{4} X_{2} Y_{2}^{7} Z_{2}+2^{5} \cdot 7^{4} Y_{2}^{8} Z_{2}+2^{3} \cdot 7^{4} X_{2}^{3} Y_{2}^{4} Z_{2}^{2}-2^{3} \cdot 7^{5} X_{2}^{2} Y_{2}^{5} Z_{2}^{2} \\
& +2^{3} \cdot 7^{4} X_{2} Y_{2}^{6} Z_{2}^{2}+2^{3} \cdot 5 \cdot 7^{4} Y_{2}^{7} Z_{2}^{2}+2^{6} \cdot 7^{4} \cdot X_{2}^{3} Y_{2}^{3} Z_{2}^{3}-2^{4} 7^{5} X_{2}^{2} Y_{2}^{4} Z_{2}^{3}-2^{3} \cdot 3^{2} \cdot 7^{4} X_{2} Y_{2}^{5} Z_{2}^{3}+2^{5} \cdot 7^{5} Y_{2}^{6} Z_{2}^{3} \\
& +2^{3} \cdot 7^{4} X_{2}^{3} Y_{2}^{2} Z_{2}^{4}-2^{4} \cdot 7^{5} X_{2}^{2} Y_{2}^{3} Z_{2}^{4}-2 \cdot 7^{4} \cdot 19 X_{2} Y_{2}^{4} Z_{2}^{4}+2 \cdot 7^{4} \cdot 97 Y_{2}^{5} Z_{2}^{4}+2^{5} \cdot 7^{4} X_{2}^{3} Y_{2} Z_{2}^{5}-2^{3} \cdot 7^{5} X_{2}^{2} Y_{2}^{2} Z_{2}^{5} \\
& -2^{3} \cdot 3^{2} \cdot 7^{4} X_{2} Y_{2}^{3} Z_{2}^{5}+2 \cdot 3^{2} \cdot 7^{4} \cdot 17 Y_{2}^{4} Z_{2}^{5}-2^{4} 37^{4} X_{2}^{2} Y_{2} Z_{2}^{6}+2^{3} \cdot 7^{4} X_{2} Y_{2}^{2} Z_{2}^{6}+2^{3} \cdot 7^{5} Y_{2}^{3} Z_{2}^{6}-2^{4} \cdot 7^{4} X_{2} Y_{2} Z_{2}^{7} \\
& +2^{3} \cdot 3 \cdot 5 \cdot 7^{4} Y_{2}^{2} Z_{2}^{7} \\
& \varphi_{1}\left(Y_{2}\right)=-2^{7} \cdot 7^{6} X_{2}^{3} Y_{2}^{6}+2^{8} \cdot 7^{6} X_{2}^{2} Y_{2}^{7}-2^{7} \cdot 7^{6} X_{2} Y_{2}^{8}+2^{6} \cdot 3 \cdot 7^{6} X_{2} Y_{2}^{7} Z_{2}-2^{6} \cdot 3 \cdot 7^{6} Y_{2}^{8} Z_{2}-2^{5} \cdot 7^{6} \cdot 11 X_{2}^{3} Y_{2}^{4} Z_{2}^{2} \\
& +2^{5} \cdot 7^{6} \cdot 29 X_{2}^{2} Y_{2}^{5} Z_{2}^{2}-2^{5} \cdot 7^{6} \cdot 11 X_{2} Y_{2}^{6} Z_{2}^{2}-2^{5} \cdot 7^{5} \cdot 127 Y_{2}^{7} Z_{2}^{2}+2^{6} \cdot 3 \cdot 7^{6} X_{2}^{3} Y_{2}^{3} Z_{2}^{3}-2^{6} \cdot 7^{6} \cdot X_{2}^{2} Y_{2}^{4} Z_{2}^{3} \\
& -2^{5} \cdot 3 \cdot 7^{6} X_{2} Y_{2}^{5} Z_{2}^{3}-2^{5} \cdot 5 \cdot 7^{5} \cdot 13 Y_{2}^{6} Z_{2}^{3}-2^{5} \cdot 7^{6} \cdot 11 X_{2}^{3} Y_{2}^{2} Z_{2}^{4}+2^{9} \cdot 7^{6} \cdot X_{2}^{2} Y_{2}^{3} Z_{2}^{4}-2^{3} \cdot 7^{6} \cdot 67 X_{2} Y_{2}^{4} Z_{2}^{4} \\
& -2^{3} \cdot 3^{3} 7^{5} \cdot 13 Y_{2}^{5} Z_{2}^{4}+2^{6} \cdot 3 \cdot 7^{6} X_{2}^{3} Y_{2} Z_{2}^{5}-2^{5} \cdot 3 \cdot 7^{6} X_{2}^{2} Y_{2}^{2} Z_{2}^{5}+2^{4} \cdot 5 \cdot 7^{6} X_{2} Y_{2}^{3} Z_{2}^{5}+2^{3} \cdot 7^{5} \cdot 167 Y_{2}^{4} Z_{2}^{5} \\
& -2^{7} \cdot 7^{6} X_{2}^{3} Z_{2}^{6}+2^{6} \cdot 7^{6} X_{2}^{2} Y_{2} Z_{2}^{6}-2^{5} \cdot 3 \cdot 7^{6} X_{2} Y_{2}^{2} Z_{2}^{6}-2^{7} \cdot 3 \cdot 7^{5} 13 Y_{2}^{3} Z_{2}^{6}+2^{7} \cdot 7^{6} \cdot X_{2}^{2} Z_{2}^{7}+2^{6} \cdot 7^{5} \cdot 41 Y_{2}^{2} Z_{2}^{7} \\
& -2^{6} \cdot 7^{5} \cdot 13 Y_{2} Z_{2}^{8} \\
& \varphi_{1}\left(Z_{2}\right)=Y_{2}^{7} Z_{2}^{2}-3 / 2 Y_{2}^{6} Z_{2}^{3}+15 / 4 Y_{2}^{5} Z_{2}^{4}+1 / 4 Y_{2}^{4} Z_{2}^{5}+15 / 4 Y_{2}^{3} Z_{2}^{6}-3 / 2 Y_{2}^{2} Z_{2}^{7}+Y_{2} Z_{2}^{8} \text {. }
\end{aligned}
$$

Then define

$$
\varphi\left(X_{2}: Y_{2}: 1\right)=\left(1 /\left(2^{6} 7^{4}\right) \varphi_{1}\left(X_{2}\right): 1 /\left(2^{9} 7^{6}\right) \varphi_{1}\left(Y_{2}\right)-1 /\left(2^{8} 7^{4}\right) \varphi_{1}\left(X_{2}\right)+3^{2} /\left(2^{2} 7\right): 1\right) .
$$

By direct checking with MAGMA $\varphi$ is an isogeny between the two elliptic curves over the field of rationals. Clearly this map provides an isogeny over $\mathbb{F}_{p}$ for every $p$ such that no coefficients of $\varphi_{1}$ vanish. It has been checked with MAGMA that also for these special values of $p$ the two elliptic curves are $\mathbb{F}_{p}$-isogenous. 
Proposition 2.3. The Jacobian variety of the curve $\mathcal{C} /\left\langle\tau_{1} \tau_{2}\right\rangle$ is $\mathbb{F}_{p}$-isogenous to twice the Jacobian variety of the elliptic curve of equation

$$
Y^{2}=X^{3}+\frac{9}{16} X^{2}+\frac{3}{16} X+\frac{1}{64} .
$$

Proof. Note that

$$
x_{3}=x(-x+1)+y(-y+1), \quad y_{3}=\frac{x}{y}+\frac{-x+1}{-y+1}
$$

are both fixed by the involution $\tau_{1} \tau_{2}$. They satisfy $L(u, v)=0$, where

$$
\begin{aligned}
L\left(x_{3}, y_{3}\right):= & 16 x_{3}^{6} y_{3}^{4}-40 x_{3}^{5} y_{3}^{4}-32 x_{3}^{5} y_{3}^{3}+32 x_{3}^{5} y_{3}^{2}+32 x_{3}^{4} y_{3}^{6}-80 x_{3}^{4} y_{3}^{5}+185 x_{3}^{4} y_{3}^{4}+80 x_{3}^{4} y_{3}^{3}+128 x_{3}^{4} y_{3}^{2} \\
& -32 x_{3}^{4} y_{3}+16 x_{3}^{4}-40 x_{3}^{3} y_{3}^{6}-124 x_{3}^{3} y_{3}^{5}-24 x_{3}^{3} y_{3}^{4}-482 x_{3}^{3} y_{3}^{3}-518 x_{3}^{3} y_{3}^{2}-112 x_{3}^{3} y_{3}-104 x_{3}^{3} \\
& +16 x_{3}^{2} y_{3}^{8}-48 x_{3}^{2} y_{3}^{7}+212 x_{3}^{2} y_{3}^{6}-352 x_{3}^{2} y_{3}^{5}+1184 x_{3}^{2} y_{3}^{4}-916 x_{3}^{2} y_{3}^{3}+2193 x_{3}^{2} y_{3}^{2}+318 x_{3}^{2} y_{3} \\
& +425 x_{3}^{2}-32 x_{3} y_{3}^{7}+48 x_{3} y_{3}^{6}-296 x_{3} y_{3}^{5}+304 x_{3} y_{3}^{4}-1076 x_{3} y_{3}^{3}+524 x_{3} y_{3}^{2}-1312 x_{3} y_{3} \\
& -832 x_{3}+32 y_{3}^{6}-64 y_{3}^{5}+308 y_{3}^{4}-488 y_{3}^{3}+1000 y_{3}^{2}-1024 y_{3}+1024 .
\end{aligned}
$$

To show that $\overline{\mathbb{F}}_{p}\left(x_{3}, y_{3}\right)$ with $L\left(x_{3}, y_{3}\right)=0$ is the function field of $\mathcal{C} /\left\langle\tau_{1} \tau_{2}\right\rangle$ it is sufficient to note that $\overline{\mathbb{F}}_{p}\left(x_{3}, y_{3}\right)$ has genus 2 . Indeed, from the Hurwitz genus formula and Theorem 1.2 applied with respect to $G, \mathcal{C} /\left\langle\sigma \tau_{1}\right\rangle$ has genus 1 and since $\tau_{1} \tau_{2} \in\left\langle\sigma \tau_{1}\right\rangle$,

$$
g\left(\mathcal{C} /\left\langle\sigma \tau_{1}\right\rangle\right)<g\left(\mathcal{C} /\left\langle\tau_{1} \tau_{2}\right\rangle\right) \leq 2 .
$$

Also, $\overline{\mathbb{F}}_{p}\left(x_{3}, y_{3}\right)$ is $\mathbb{F}_{p}$-isomorphic to $\overline{\mathbb{F}}_{p}\left(u^{\prime}, v^{\prime}\right)$, with

$$
v^{\prime 2}+\left(u^{\prime 2}+u^{\prime}\right) v^{\prime}=-u^{\prime 6}-3{u^{\prime}}^{5}-u^{\prime 4}-7 u^{\prime 3}-u^{\prime 2}-3 u^{\prime}-1
$$

via the isomorphism $\phi\left(x_{3}, y_{3}\right)=\left(\frac{b_{1}\left(x_{3}, y_{3}\right)}{b_{3}\left(x_{3}, y_{3}\right)}, \frac{b_{2}\left(x_{3}, y_{3}\right)}{b_{3}\left(x_{3}, y_{3}\right)}\right)$ given by

$$
\begin{aligned}
& b_{1}\left(x_{3}, y_{3}\right)=-2 x_{3}^{4}+x_{3}^{3}-2 x_{3}^{2}, \\
& b_{2}\left(x_{3}, y_{3}\right)=\frac{2 x_{3}^{5}-3 x_{3}^{4}+x_{3}^{3}-2 x_{3}^{2} y_{3}-3 x_{3}^{2}-x_{3} y_{3}-y_{3}-1}{2}, \\
& b_{3}\left(x_{3}, y_{3}\right)=x_{3}^{5}+2 x_{3}^{3}+x_{3} .
\end{aligned}
$$

Now, $\overline{\mathbb{F}}_{p}\left(u^{\prime}, v^{\prime}\right)$ is $\mathbb{F}_{p}$-isomoprhic to $\overline{\mathbb{F}}_{p}\left(u^{\prime \prime}, v^{\prime \prime}\right)$, with

$$
\left(v^{\prime \prime}\right)^{2}=-4\left(u^{\prime \prime}\right)^{6}-12\left(u^{\prime \prime}\right)^{5}-3\left(u^{\prime \prime}\right)^{4}-26\left(u^{\prime \prime}\right)^{3}-3\left(u^{\prime \prime}\right)^{2}-12\left(u^{\prime \prime}\right)-4,
$$

via the isomorphism $\theta\left(u^{\prime}, v^{\prime}\right)=\left(\frac{c_{1}\left(u^{\prime}, v^{\prime}\right)}{c_{3}\left(u^{\prime}, v^{\prime}\right)}, \frac{c_{2}\left(u^{\prime}, v^{\prime}\right)}{c_{3}\left(u^{\prime}, v^{\prime}\right)}\right)$

$$
\begin{aligned}
& c_{1}\left(u^{\prime}, v^{\prime}\right)=u^{\prime 2}, \\
& c_{2}\left(u^{\prime}, v^{\prime}\right)=u^{\prime 2}+u^{\prime}+2 v^{\prime}, \\
& c_{3}\left(u^{\prime}, v^{\prime}\right)=u^{\prime 3} .
\end{aligned}
$$


Consider the involutory automorphisms of the hyperelliptic function field $\overline{\mathbb{F}}_{p}\left(u^{\prime \prime}, v^{\prime \prime}\right)$ given by

$$
\psi_{1}\left(u^{\prime \prime}, v^{\prime \prime}\right)=\left(1 / u^{\prime \prime}, v^{\prime \prime} /\left(u^{\prime \prime}\right)^{3}\right) \quad \psi_{2}\left(u^{\prime \prime}, v^{\prime \prime}\right)=\left(u^{\prime \prime},-v^{\prime \prime}\right) .
$$

The fixed fields of $\overline{\mathbb{F}}_{p}\left(u^{\prime \prime}, v^{\prime \prime}\right)$ with respect to $\psi_{1}, \psi_{2}, \psi_{1} \psi_{2}$ are

$$
\overline{\mathbb{F}}_{p}\left(s, t_{1}\right), \overline{\mathbb{F}}_{p}\left(u^{\prime \prime}\right), \overline{\mathbb{F}}_{p}\left(s, t_{2}\right),
$$

respectively, where $s=u^{\prime \prime}+1 / u^{\prime \prime}, t_{1}=v^{\prime \prime}+v^{\prime \prime} /\left(u^{\prime \prime}\right)^{3}, t_{2}=v^{\prime \prime}-v^{\prime \prime} /\left(u^{\prime \prime}\right)^{3}$ and

$$
\begin{aligned}
& t_{1}^{2}-4 s^{6}-12 s^{5}+21 s^{4}+26 s^{3}-51 s^{2}+24 s-4=0, \\
& -t_{2}^{2}-4 s^{6}-12 s^{5}+21 s^{4}+42 s^{3}-3 s^{2}-12 s+4=0 .
\end{aligned}
$$

Consider the function fields $\overline{\mathbb{F}}_{p}\left(s^{\prime}, t_{1}^{\prime}\right), \overline{\mathbb{F}}_{p}\left(s^{\prime \prime}, t_{1}^{\prime \prime}\right)$ with

$$
\left(t_{1}^{\prime}\right)^{2}=\left(s^{\prime}\right)^{3}-9517824 s^{\prime}+11448262656
$$

and

$$
\left(t_{2}^{\prime}\right)^{2}=\left(s^{\prime \prime}\right)^{3}-4541184\left(s^{\prime \prime}\right)+493881753 .
$$

The function field $\overline{\mathbb{F}}_{p}\left(s, t_{1}\right)$ is isomorphic to $\overline{\mathbb{F}}_{p}\left(s^{\prime}, t_{1}^{\prime}\right)$ via the isomorphism

$$
\theta^{\prime}\left(s, t_{1}\right)=\left(d_{1}\left(s, t_{1}\right) / d_{3}\left(s, t_{1}\right), d_{2}\left(s, t_{1}\right) / d_{3}\left(s, t_{1}\right)\right)
$$

where

$$
\begin{aligned}
d_{1}\left(s, t_{1}\right)= & 2304 s^{5}+9216 u^{4}-2880 s^{3}+1152 s^{2} t_{1}-17856 s^{2}+2880 s t_{1}+11520 s+720 t_{1}-2304 \\
d_{2}\left(s, t_{1}\right)= & 248832 s^{5}+995328 s^{4}+55296 s^{3} t_{1}-311040 s^{3}+207360 s^{2} t_{1}-1928448 s^{2}+103680 s t_{1} \\
& +27648 t_{1}^{2}+1244160 s-55296 t_{1}-248832 \\
d_{3}\left(s, t_{1}\right)= & t_{1} .
\end{aligned}
$$

The function field $\overline{\mathbb{F}}_{p}\left(s, t_{2}\right)$ is isomorphic to $\overline{\mathbb{F}}_{p}\left(s^{\prime \prime}, t_{2}^{\prime}\right)$ via the isomorphism

$$
\theta^{\prime \prime}\left(s, t_{2}\right)=\left(d_{1}^{\prime}\left(s, t_{2}\right) / d_{3}^{\prime}\left(s, t_{2}\right), d_{2}^{\prime}\left(s, t_{2}\right) / d_{3}^{\prime}\left(s, t_{2}\right)\right)
$$

where

$$
\begin{aligned}
d_{1}^{\prime}\left(s, t_{2}\right)= & 36864 s^{5} t_{2}+184320 s^{4} t_{2}+175104 s^{3} t_{2}-36864 s^{2} t_{2}-4176 t_{2}^{3}-46080 s t_{2}+18432 t_{2} \\
d_{2}^{\prime}\left(s, t_{2}\right)= & 84934656 s^{5}-442368 s^{3} t_{2}^{2}+424673280 s^{4}-2654208 s^{2} t_{2}^{2}+403439616 s^{3}-5640192 s t_{2}^{2} \\
& -84934656 s^{2}-10506240 t_{2}^{2}-106168320 s+42467328 \\
d_{3}^{\prime}\left(s, t_{2}\right)= & t_{2}^{3} .
\end{aligned}
$$


The two elliptic curves $\mathcal{E}_{1}: Y^{2}=X^{3}-9517824 X+11448262656$ and $\mathcal{E}_{2}: Y^{2}=X^{3}-4541184 X+$ 493881753 are isogenous to $Y^{2}=X^{3}+9 / 16 X^{2}+3 / 16 X+1 / 64$ via the $\mathbb{F}_{p^{2}}$-isogenies

where

$$
(X: Y) \mapsto\left(\frac{f_{1}(X)}{f_{2}(X)}: \frac{g(X, Y)}{f_{3}(X)}\right)
$$

$$
\begin{aligned}
& f_{1}(X)=-1 / 20736 X^{3}-1 / 16 X^{2}+837 X-1123632 \\
& f_{2}(X)=X^{2}-2592 X+1679616 \\
& g(X, Y)=-\xi / 2985984 X^{3} Y+\xi / 768 X^{2} Y-75 \xi / 16 X Y+8073 \xi Y, \\
& f_{3}(X)=-X^{3}+3888 X^{2}-5038848 X+2176782336
\end{aligned}
$$

with $\xi^{2}=-1$ for $\mathcal{E}_{1}$, and

$$
\begin{aligned}
& f_{1}(X)=1 / 20736 X^{3}-11 / 48 X^{2}-213 X+720720, \\
& f_{2}(X)=X^{2}-864 X+186624 \\
& g(X, Y)=1 / 2985984 X^{3} Y-1 / 2304 X^{2} Y+137 / 48 X Y-9371 Y, \\
& f_{3}(X)=X^{3}-1296 X^{2}+559872 X-80621568
\end{aligned}
$$

for $\mathcal{E}_{2}$. From Corollary 1.2 applied to $\overline{\mathbb{F}}_{p}\left(u^{\prime \prime}, v^{\prime \prime}\right)$ with respect to $\left\langle\psi_{1}, \psi_{2}\right\rangle \cong C_{2} \times C_{2}$ the claim follows.

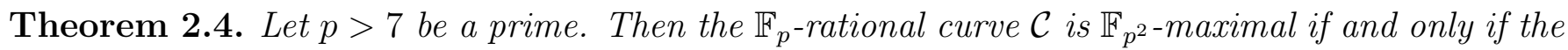
elliptic curve

$$
\mathcal{E}: Y^{2}=X^{3}+9 / 16 X^{2}+3 / 16 X+1 / 64
$$

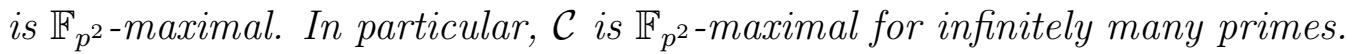

Proof. We apply Theorem 1.1 to the curve $\mathcal{C}$ and the group $G=\left\langle\tau_{1}, \tau_{2}, \sigma\right\rangle$. Such a group can be written as

$$
G=\left\langle\tau_{1}\right\rangle \cup\left\langle\tau_{2}\right\rangle \cup\langle\sigma\rangle \cup\left\langle\sigma \tau_{1}\right\rangle \cup\left\langle\sigma \tau_{1} \tau_{2}\right\rangle ;
$$

see Equation (2). In this case, $m=5, h_{1}=h_{2}=h_{3}=h_{5}=2, h_{4}=4$ and

$$
J_{\mathcal{C}}^{4} \times J_{\mathcal{C} / G}^{4} \simeq J_{\mathcal{C} /\left\langle\tau_{1}\right\rangle}^{2} \times J_{\mathcal{C} /\left\langle\tau_{2}\right\rangle}^{2} \times J_{\mathcal{C} /\langle\sigma\rangle}^{2} \times J_{\mathcal{C} /\left\langle\sigma \tau_{1}\right\rangle}^{4} \times J_{\mathcal{C} /\left\langle\sigma \tau_{1} \tau_{2}\right\rangle}^{2} .
$$

By the Hurwitz genus formula applied to $G$, we deduce that $\mathcal{C} / G$ is rational. In fact, the number of places of $\mathcal{C}$ fixed by elemets of $G$ exceeds 6. By Propositions 2.1 and 2.2 the Jacobians of $\mathcal{C} /\left\langle\tau_{1}\right\rangle$, $\mathcal{C} /\left\langle\tau_{2}\right\rangle, \mathcal{C} /\langle\sigma\rangle$ and $\mathcal{C} /\left\langle\sigma \tau_{1}\right\rangle$ are $\mathbb{F}_{p}$-isogenous to a power of the Jacobian of $\mathcal{E}$. Hence Equation (77) reads

$$
J_{\mathcal{C}} \simeq J_{\mathcal{E}}^{3} \times J_{\mathcal{C} /\left\langle\sigma \tau_{1}\right\rangle}
$$

To prove the first claim, note that the $\mathbb{F}_{p^{2}}$-maximality of $\mathcal{C}$ implies the $\mathbb{F}_{p^{2}}$-maximality of $\mathcal{E}$ since $\mathcal{E}$

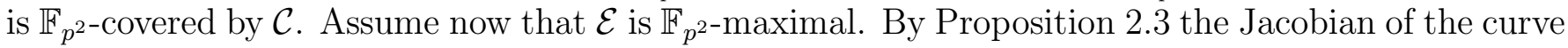
$\mathcal{C} /\left\langle\tau_{1} \tau_{2}\right\rangle$ is $\mathbb{F}_{p^{2}}$-isogenous to a power of $J_{\mathcal{E}}$, and hence $\mathcal{C} /\left\langle\tau_{1} \tau_{2}\right\rangle$ is an $\mathbb{F}_{p^{2}}$-maximal curve. But $\mathcal{C} /\left\langle\sigma \tau_{1}\right\rangle$

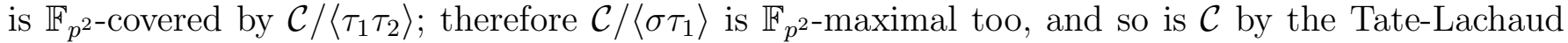
criterion mentioned in the introduction. 
An elliptic curve over the complex field with integer coefficients, when it is viewed as a curve defined over $\mathbb{F}_{p}$ with $p$ a prime, is supersingular for infinitely many $p$ 's; see [19]. Since a supersingular elliptic curve defined over $\mathbb{F}_{p}$ is $\mathbb{F}_{p^{2}}$-maximal, the elliptic curve $\mathcal{E}$ is $\mathbb{F}_{p^{2}}$-maximal for infinitely many $p$ 's. This proves the second claim.

Remark 2.5. MAGMA [9] computations show that the list of primes up to 100000 for which the curve $\mathcal{E}$, and therefore $\mathcal{C}$, is $\mathbb{F}_{p^{2}}$-maximal is

$17,71,251,647,827,1889,3527,3617,4409,6569,11969,12113,12527,12689,13913,22031$,

23039, 23633, 26297, 28871, 31769, 35171, 35729, 48527, 60497, 60623, 61487, 82457, 93383, 93761.

Remark 2.6. For $p=17$ the curve $\mathcal{C}$ is Galois covered by the Hermitian curve $\mathcal{H}_{17}$ over $\mathbb{F}_{17^{2}}$. In fact, let $\mathcal{H}_{17}: Y^{18}=X^{17} Z+Z^{17} X$. Consider the automorphism group $G$ of $\mathcal{H}_{17}$ generated by $\alpha_{1}, \alpha_{2}, \alpha_{3}$ and $\gamma$ where

$$
\begin{gathered}
\alpha_{1}(X, Y, Z)=(-X, \lambda Y, Z), \alpha_{2}(X, Y, Z)=(-\lambda c Z, Y,-\lambda / c X), \\
\alpha_{3}(X, Y, Z)=(c X, Y,-X / c), \text { and } \gamma(X, Y, Z)=(X, \mu Y, Z),
\end{gathered}
$$

with $\mu^{3}=1, \lambda^{2}=-1$ and $2 c^{2}=\lambda+1$. Then $G=\left\langle\alpha_{1}, \alpha_{2}, \alpha_{3}\right\rangle \times\langle\gamma\rangle \cong Q_{8} \times C_{3}$, where $Q_{8}$ is the quaternion group of order 8. Using MAGMA we obtained a model for the quotient curve $\mathcal{H}_{17} / G$ over $\mathbb{F}_{17^{2}}$ :

$$
\mathcal{H}_{17} / G:\left\{\begin{array}{l}
Y Z+16 X W=0 \\
X^{3}+4 X Y^{2}+16 Z^{2} W+9 W^{3}=0
\end{array}\right.
$$

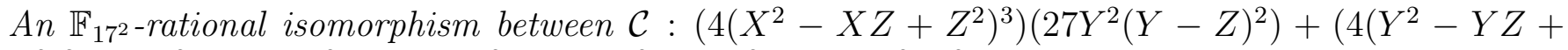
$\left.\left.Z^{2}\right)^{3}\right)\left(27 X^{2}(X-Z)^{2}\right)=\left(27 Y^{2}(Y-Z)^{2}\right)\left(27 X^{2}(X-Z)^{2}\right) Z^{2}$ and $\mathcal{H}_{17} / G$ is given by $\phi(X, Y, Z)=$ $\left(\phi_{1}(X, Y, Z), \phi_{2}(X, Y, Z), \phi_{3}(X, Y, Z), \phi_{4}(X, Y, Z)\right)$ where

$$
\begin{gathered}
\phi_{1}(X, Y, Z)=w^{159} X^{5} Y^{6} Z+w^{159} X^{3} Y^{8} Z+w^{33} X^{5} Y^{5} Z^{2}+w^{28} X^{4} Y^{6} Z^{2}+w^{231} X^{3} Y^{7} Z^{2}+w^{89} X^{2} Y^{8} Z^{2} \\
+w^{141} X^{5} Y^{4} Z^{3}+w^{190} X^{4} Y^{5} Z^{3}+w^{78} X^{3} Y^{6} Z^{3}+w^{161} X^{2} Y^{7} Z^{3}+w^{93} X Y^{8} Z^{3}+w^{213} X^{5} Y^{3} Z^{4}+w^{135} X^{4} Y^{4} Z^{4} \\
+2 X^{3} Y^{5} Z^{4}+4 X^{2} Y^{6} Z^{4}+w^{165} X Y^{7} Z^{4}+w^{156} Y^{8} Z^{4}+w^{177} X^{5} Y^{2} Z^{5}+w^{253} X^{4} Y^{3} Z^{5}+w^{119} X^{3} Y^{4} Z^{5} \\
+w^{91} X^{2} Y^{5} Z^{5}+w^{127} X Y^{6} Z^{5}+w^{228} Y^{7} Z^{5}+w^{124} X^{4} Y^{2} Z^{6}+w^{281} X^{3} Y^{3} Z^{6}+X^{2} Y^{4} Z^{6}+w^{52} X Y^{5} Z^{6} \\
+14 Y^{6} Z^{6}+w^{209} X^{3} Y^{2} Z^{7}+w^{101} X^{2} Y^{3} Z^{7}+w^{64} X Y^{4} Z^{7}+w^{64} Y^{5} Z^{7}+w^{69} X^{3} Z^{8}+w^{194} X^{2} Y^{2} Z^{8} \\
+w^{221} X Y^{3} Z^{8}+w^{275} Y^{4} Z^{8}+w^{177} X^{3} Z^{9}+w^{74} X^{2} Y Z^{9}+w^{97} X Y^{2} Z^{9}+w^{220} Y^{3} Z^{9}+3 X^{2} Z^{10}+w^{75} X Y Z^{10} \\
+w^{202} Y^{2} Z^{10}+w^{129} X Z^{11}+w^{12} Y Z^{11}
\end{gathered}
$$

$$
\begin{aligned}
& \phi_{2}(X, Y, Z)=w^{105} X^{5} Y^{7}+w^{105} X^{3} Y^{9}+w^{195} X^{5} Y^{6} Z+w^{52} X^{4} Y^{7} Z+w^{33} X^{3} Y^{8} Z+12 X^{2} Y^{9} Z+w^{267} X^{5} Y^{5} Z^{2} \\
& +w^{142} X^{4} Y^{6} Z^{2}+w^{94} X^{3} Y^{7} Z^{2}+14 X^{2} Y^{8} Z^{2}+w^{57} X Y^{9} Z^{2}+w^{51} X^{5} Y^{4} Z^{3}+w^{214} X^{4} Y^{5} Z^{3}+w^{132} X^{3} Y^{6} Z^{3} \\
& +w^{76} X^{2} Y^{7} Z^{3}+w^{273} X Y^{8} Z^{3}+w^{231} X^{5} Y^{3} Z^{4}+w^{286} X^{4} Y^{4} Z^{4}+w^{38} X^{3} Y^{5} Z^{4}+w^{286} X^{2} Y^{6} Z^{4}+w^{128} X Y^{7} Z^{4} \\
& +w^{285} X^{5} Y^{2} Z^{5}+w^{178} X^{4} Y^{3} Z^{5}+w^{8} X^{3} Y^{4} Z^{5}+w^{75} X^{2} Y^{5} Z^{5}+w^{207} X Y^{6} Z^{5}+w^{165} Y^{7} Z^{5}+w^{232} X^{4} Y^{2} Z^{6} \\
& +w^{175} X^{3} Y^{3} Z^{6}+w^{187} X^{2} Y^{4} Z^{6}+w^{231} X Y^{5} Z^{6}+w^{255} Y^{6} Z^{6}+w^{212} X^{3} Y^{2} Z^{7}+w^{145} X^{2} Y^{3} Z^{7}+w^{179} X Y^{4} Z^{7} \\
& \quad+w^{39} Y^{5} Z^{7}+w^{123} X^{3} Y Z^{8}+w^{71} X^{2} Y^{2} Z^{8}+w^{151} X Y^{3} Z^{8}+w^{111} Y^{4} Z^{8}+w^{285} X^{3} Z^{9}+2 X^{2} Y Z^{9}
\end{aligned}
$$




$$
+w^{82} X Y^{2} Z^{9}+w^{3} Y^{3} Z^{9}+11 X^{2} Z^{10}+w^{75} X Y Z^{10}+w^{57} Y^{2} Z^{10}+w^{237} X Z^{11},
$$

$$
\begin{gathered}
\phi_{3}(X, Y, Z)=w^{207} X^{5} Y^{7}+w^{207} X^{3} Y^{9}+w^{9} X^{5} Y^{6} Z+w^{189} X^{4} Y^{7} Z+w^{135} X^{3} Y^{8} Z+w^{117} X^{2} Y^{9} Z+w^{45} X^{5} Y^{5} Z^{2} \\
+w^{279} X^{4} Y^{6} Z^{2}+w^{225} X^{3} Y^{7} Z^{2}+w^{45} X^{2} Y^{8} Z^{2}+w^{243} X Y^{9} Z^{2}+w^{45} X^{5} Y^{4} Z^{3}+w^{27} X^{4} Y^{5} Z^{3}+w^{171} X^{3} Y^{6} Z^{3} \\
+w^{27} X^{2} Y^{7} Z^{3}+w^{171} X Y^{8} Z^{3}+w^{9} X^{5} Y^{3} Z^{4}+w^{27} X^{4} Y^{4} Z^{4}+w^{27} X^{3} Y^{5} Z^{4}+w^{207} X^{2} Y^{6} Z^{4}+w^{135} X Y^{7} Z^{4} \\
+w^{207} X^{5} Y^{2} Z^{5}+w^{279} X^{4} Y^{3} Z^{5}+w^{27} X^{3} Y^{4} Z^{5}+w^{9} X^{2} Y^{5} Z^{5}+w^{9} X Y^{6} Z^{5}+w^{167} Y^{7} Z^{5}+w^{189} X^{4} Y^{2} Z^{6} \\
\quad+w^{171} X^{3} Y^{3} Z^{6}+w^{9} X^{2} Y^{4} Z^{6}+w^{207} X Y^{5} Z^{6}+w^{257} Y^{6} Z^{6}+w^{225} X^{3} Y^{2} Z^{7}+w^{207} X^{2} Y^{3} Z^{7} \\
+w^{207} X Y^{4} Z^{7}+w^{136} Y^{5} Z^{7}+w^{135} X^{3} Y Z^{8}+w^{27} X^{2} Y^{2} Z^{8}+w^{9} X Y^{3} Z^{8}+w^{171} Y^{4} Z^{8}+w^{207} X^{3} Z^{9}+w^{45} X^{2} Y Z^{9} \\
+w^{135} X Y^{2} Z^{9}+w^{257} Y^{3} Z^{9}+w^{117} X^{2} Z^{10}+w^{171} X Y Z^{10}+w^{140} Y^{2} Z^{10}+w^{243} X Z^{11}+4 Y Z^{11},
\end{gathered}
$$$$
\phi_{4}(X, Y, Z)=Y^{8} Z^{4}+13 Y^{7} Z^{5}+Y^{6} Z^{6}+11 Y^{5} Z^{7}+5 Y^{4} Z^{8}+Y^{3} Z^{9}+10 Y^{2} Z^{10}+9 Y Z^{11}
$$

and $w$ is a primitive element of $\mathbb{F}_{17^{2}}$. We are not able to tell whether $\mathcal{C}$ is Galois covered by the Hermitian curve for $p>17$.

2.2. Automorphism group of $\mathcal{C}$. The aim of this section is to compute the full automorphism group of $\mathcal{C}$ when $p \geq 5$. First, we construct a subgroup $H$ of automorphisms of $\mathcal{C}$, and then we will prove that when $p \geq 5, H$ coincides with the full automorphism group of $\mathcal{C}$.

Remark 2.7. The full automorphism group of $\mathcal{C}$ contains a subgroup

$$
H=\left(\left\langle\alpha_{1}, \alpha_{2}\right\rangle \times\left\langle\alpha_{3}, \alpha_{4}\right\rangle\right) \rtimes\left\langle\alpha_{5}\right\rangle \cong\left(S_{3} \times S_{3}\right) \rtimes C_{2},
$$

where

$$
\begin{gathered}
\alpha_{1}:(x, y) \mapsto\left(\frac{-1}{x-1}, y\right), \quad \alpha_{2}:(x, y) \mapsto\left(\frac{1}{x}, y\right) \\
\alpha_{3}:(x, y) \mapsto\left(x, \frac{-1}{y-1}\right), \quad \alpha_{4}:(x, y) \mapsto\left(x, \frac{1}{y}\right), \quad \alpha_{5}:(x, y) \mapsto(y, x) .
\end{gathered}
$$

It is easily seen that

$$
\alpha_{2}^{2}=\alpha_{4}^{2}=\alpha_{5}^{2}=\alpha_{1}^{3}=\alpha_{3}^{3}=1
$$

and $\alpha_{2} \alpha_{1} \alpha_{2}=\alpha_{1}^{2}, \alpha_{4} \alpha_{3} \alpha_{4}=\alpha_{3}^{2}$ so that $\left\langle\alpha_{1}, \alpha_{2}\right\rangle \cong \mathrm{S}_{3}$ and $\left\langle\alpha_{3}, \alpha_{4}\right\rangle \cong \mathrm{S}_{3}$, and $\alpha_{5}$ interchanges $\left\langle\alpha_{1}, \alpha_{2}\right\rangle$ into $\left\langle\alpha_{3}, \alpha_{4}\right\rangle$ by conjugation.

Lemma 2.8. $|A u t(\mathcal{C})| \in\{72,144,216\}$. 
Proof. We first prove that the size of $A u t(\mathcal{C})$ is coprime with $p$. Assume on the contrary that $S$ is a Sylow $p$-subgroup of $\operatorname{Aut}(\mathcal{C})$. Then by [65, Theorem 1],

$$
|S| \leq \max \left\{4, \frac{4 p}{(p-1)^{2}} 16\right\} \text {. }
$$

For $p \geq 11$ this contradicts $|S| \geq p$. By direct checking with MAGMA, if either $p=5$ or $p=7$ then the curve $\mathcal{C}$ is ordinary, that is, the $p$-rank $\gamma$ of $\mathcal{C}$ equals its genus $g(\mathcal{C})=4$. If $p=7$, by [65, Theorem 1 (i)] we have $|S| \leq 3 p /(p-2) \leq 21 / 5<7$, a contradiction. If $p=5$ then $|S|=5$. By [37, Theorem 1.3], $|A u t(\mathcal{C})|$ divides $2 p(p-1)=40$, a contradiction as 72||$A u t(\mathcal{C}) \mid$ by Remark 2.7. This shows that $p$ does not divide $|A u t(\mathcal{C})|$. By [46, Theorem 11.108], $|A u t(\mathcal{C})|=2^{a} 3^{b} 5^{c} 7^{d}$ for some integers $a, b, c, d \geq 0$. Observe that $a \geq 3$ and $b \geq 2$ as 72||$A u t(\mathcal{C}) \mid$. Hurwitz bound [46, Theorem 11.56] yields $|A u t(\mathcal{C})| \leq 84(g-1)=252$, hence $|A u t(\mathcal{C})| \in\{72,144,216\}$.

Next we prove that the cases $|A u t(\mathcal{C})|=144$ and $|A u t(\mathcal{C})|=216$ cannot actually occur.

Theorem 2.9. Let $p \geq 5$ and let $H$ be as in Remark 2.7. The full automorphism group Aut $(\mathcal{C})$ coincides with $H$. In particular, $A u t(\mathcal{C}) \cong\left(S_{3} \times S_{3}\right) \rtimes C_{2}$, where $C_{2}$ denotes a cyclic group of order 2 and $S_{3}$ is the symmetric group on 3 letters.

Proof. - Suppose that $|A u t(\mathcal{C})|=216$. Then $A u t(\mathcal{C})$ is one of the 177 groups of order 216 (up to isomorphism) and it contains a subgroup $G$ isomorphic to $\left(S_{3} \times S_{3}\right) \rtimes C_{2}$. By direct checking with MAGMA, we have that $\operatorname{Aut}(\mathcal{C}) \cong \operatorname{SmallGroup}(216, i)$ for some $i=157,158,159$. We recall that from [46, Theorem 11.79] if $A$ is an abelian subgroup of $\operatorname{Aut}(\mathcal{C})$, then $|A| \leq$ $4 g+4=20$. On the other hand, for each $i=157,158,159$ the group $\operatorname{SmallGroup}(216, i)$ has an abelian subgroup of order 27, a contradiction.

- Suppose that $|A u t(\mathcal{C})|=144$. In particular, $A u t(\mathcal{C})$ is one of the 197 groups of order 144 (up to isomorphism) containing a subgroup isomorphic to $\left(S_{3} \times S_{3}\right) \rtimes C_{2}$. By direct checking with MAGMA, Aut $(\mathcal{C}) \cong \operatorname{SmallGroup}(144, i)$ for some $i=182,186$. Since SmallGroup $(144,186)$ contains an elementary abelian group of order 8 and $\mathcal{C}$ has even genus, [36, Lemma 6.3] provides a contradiction. Hence, $\operatorname{Aut}(\mathcal{C}) \cong \operatorname{SmallGroup}(144,182)$. Therefore, $\operatorname{Aut}(\mathcal{C})$ contains a unique Sylow 3-subgroup $S_{3}$, which is elementary abelian of order 9; also, the elements of order 3 are all conjugated in $A u t(\mathcal{C})$ whereas they split into 2 conjugacy classes in the subgroup $H \cong\left(S_{3} \times S_{3}\right) \rtimes C_{2}$. Let $S$ denote the unique Sylow 3-subgroup of $A u t(\mathcal{C})$ and let $\tilde{g}$ be the genus of the quotient curve $\mathcal{C} / S$. By the Hurwitz genus formula [46, Theorem 11.72] applied to $S$, either $\tilde{g}=1$ or $\tilde{g}=0$.

Assume that $\tilde{g}=1$. Then from [46, Theorem 11.72],

$$
6=2 \cdot a+8 \cdot b
$$

for some $a \geq 0$ and $b \geq 0$ as $S$ contains just proper subgroups of order 3 . Necessarily, $b=0$ and $a=3$. Geometrically, this shows that for 3 points, say $P_{1}, P_{2}$, and $P_{3}$, the stabilizer $S_{P_{i}}$ has order 3 . Since the 8 elements of order 3 in $S$ are all conjugated and therefore fix the same number $n$ of points, the only possibility is $n=1$ and $S_{P_{i}} \neq S_{P_{j}}$ for every $i \neq j$ and $i, j=1,2,3$. Note that $S$ acts transitively on the set $\left\{P_{1}, P_{2}, P_{3}\right\}$. Let $N$ be the normalizer of $S$ in $\operatorname{Aut}(\mathcal{C})$. Then by direct checking with MAGMA, $N=S \rtimes M$, for some subgroup 
$M$ of order 16 . The group $M$ is isomorphic to a subgroup of automorphisms of the elliptic curve $\mathcal{C} / S$, which fixes the point of $\mathcal{C} / S$ corresponding to the orbit $\left\{P_{1}, P_{2}, P_{3}\right\}$. But this is impossible by [46, Theorem 11.94(ii)].

We are left with the case where $\mathcal{C} / S$ is a rational curve. Note that $S$ cannot have fixed points on $\mathcal{C}$ since it is not a cyclic group; see [46, Theorem 11.49]. By the Hurwitz genus formula we get that

$$
6=-2 \cdot 9+2 a,
$$

and hence $a=12$ and each element of order 3 in $S$ has exactly 3 fixed points. For a subgroup $T$ of $S$ of order 3, by the Hurwitz genus formula applied to $T$, the quotient curve $\mathcal{C} / T$ is elliptic. The normalizer $N_{T}$ of $T$ in $A u t(\mathcal{C})$ has order 36 and the factor group $N_{T} / T$ contains an elementary abelian subgroup $K$ of order 4 . Clearly, $N_{T}$ acts on the set of 3 points fixed by $T$. Therefore $K$, viewed as an automorphism group of $\mathcal{C} / T$, acts on the set of 3 points of $\mathcal{C} / T$ corresponding to the fixed points of $T$. Necessarily $K$ fixes at least one of these 3 points. Since $K$ is not cyclic we have a contradiction by [46, Theorem 11.49].

This shows that the elements of order 3 cannot be all conjugated in $A u t(\mathcal{C})$ and hence that $A u t(\mathcal{C})$ cannot be isomorphic to $\operatorname{SmallGroup}(144,182)$.

By Lemma 2.8 Aut $(\mathcal{C})$ has order 72 , and the claim follows by Remark 2.7.

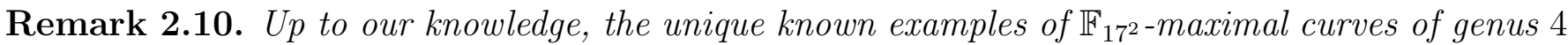
were

and

$$
y^{12}=x(x+1)^{16}
$$

$$
x^{6}+y^{6}+2 x^{2} y^{2}+12 x y+14=0 ;
$$

see [68, 88]. We point out that the curve $\mathcal{C}$ is not $\overline{\mathbb{F}}_{17}$-isomorphic to either of them, since the automorphism group of the former is $\left(\left(C_{2} \times C_{2}\right) \rtimes\left(C_{3} \times C_{3}\right)\right) \rtimes C_{2} ¥ A$ Aut $(\mathcal{C})$, whereas the order of the automorphism group of the latter is 12 .

\section{Curves of Genus 5}

Let $q=p^{h}$ where $h \in \mathbb{N}$ and $p$ is an odd prime. Let $a \in \mathbb{F}_{q^{2}}$ be such that

$$
a^{2}+108 \neq 0 \text {. }
$$

We consider the following family of algebraic curves

$$
\mathcal{C}_{a}: Y^{2}=X^{12}-a X^{10}-33 X^{8}+2 a X^{6}-33 X^{4}-a X^{2}+1 .
$$

Let $F_{a}=\overline{\mathbb{F}}_{p}(x, y)$ with $y^{2}=x^{12}-a x^{10}-33 x^{8}+2 a x^{6}-33 x^{4}-a x^{2}+1$ be the corresponding function field. Let $G=\left\langle\alpha_{1}, \alpha_{2}\right\rangle$, where $\alpha_{1}, \alpha_{2}$ are the following involutory automorphisms of $F_{a}$ :

$$
\begin{aligned}
& \alpha_{1}:(x, y) \rightarrow(x,-y), \\
& \alpha_{2}:(x, y) \rightarrow(-x, y) .
\end{aligned}
$$

Lemma 3.1. Let $q=p^{h}$ where $h \in \mathbb{N}$ and $p$ is an odd prime. Let $a \in \mathbb{F}_{q^{2}}$. Then the curve $\mathcal{C}_{a}$ defined as in (10) has genus 5 and it is hyperelliptic. 
Proof. To prove the statement it is sufficient to observe that, if $f(X)=X^{12}-a X^{10}-33 X^{8}+2 a X^{6}-$ $33 X^{4}-a X^{2}+1$ then $f(X)$ has multiple roots if and only if $a^{2}+108=0$ since the resultant of $f(X)$ and its derivative $f^{\prime}(X)$ is equal to $\left(a^{2}+108\right)^{8}$. Now the claim follows from [74, Proposition 3.7.3 and Corollary 3.7.4].

A decomposition of the Jacobian $J_{a}$ of $\mathcal{C}_{a}$ is obtained applying Theorem 1.1, with respect to the automorphism group $G=\left\langle\alpha_{1}, \alpha_{2}\right\rangle$.

Remark 3.2. Since $\alpha_{1}$ is the hyperelliptic involution of $\mathcal{C}_{a}$, the fixed fields FixG and Fix $\left\langle\alpha_{1}\right\rangle$ are both rational, see [46, Theorem 11.98]. More precisely, it is easily seen that Fix $\left\langle\alpha_{1}\right\rangle=\overline{\mathbb{F}}_{p}(x)$ and Fix $G=\overline{\mathbb{F}}_{p}\left(x^{2}\right)$.

To apply Theorem 1.1 we need to compute the fixed fields of $\left\langle\alpha_{2}\right\rangle$ and $\left\langle\alpha_{3}\right\rangle=\left\langle\alpha_{1} \alpha_{2}\right\rangle$.

Lemma 3.3. Let $q=p^{h}$ where $h \in \mathbb{N}$ and $p$ is an odd prime. Let $a \in \mathbb{F}_{q^{2}}$ with $a^{2}+108 \neq 0$. Then Fix $\left\langle\alpha_{2}\right\rangle=\overline{\mathbb{F}}_{p}(\eta, \theta)$ where $\eta=y, \theta=x^{2}$ and

$$
\eta^{2}=\theta^{6}-a \theta^{5}-33 \theta^{4}+2 a \theta^{3}-33 \theta^{2}-a \theta+1
$$

In particular Fix $\left\langle\alpha_{2}\right\rangle$ is a hyperelliptic curve of genus 2.

Proof. The first assertion follows from the fact that $x^{2}$ is fixed by $\alpha_{2}$.

The polynomial $f_{1}(X)=X^{6}-a X^{5}-33 X^{4}+2 a X^{3}-33 X^{2}-a X+1$ has no multiple roots by Lemma 3.1. From [74, Proposition 3.7.3 and Corollary 3.7.4], Fix $\left\langle\alpha_{2}\right\rangle$ is hyperelliptic of genus 2.

Lemma 3.4. Let $q=p^{h}$ where $h \in \mathbb{N}$ and $p$ is an odd prime. Let $a \in \mathbb{F}_{q^{2}}$ with $a^{2}+108 \neq 0$ and let $\alpha_{3}=\alpha_{1} \alpha_{2}$ so that $\alpha_{3}:(x, y) \rightarrow(-x,-y)$. Then Fix $\left\langle\alpha_{3}\right\rangle=\overline{\mathbb{F}}_{p}(\rho, \theta)$ where $\rho=x y, \theta=x^{2}$ and

$$
\rho^{2}=\theta^{7}-a \theta^{6}-33 \theta^{5}+2 a \theta^{4}-33 \theta^{3}-a \theta^{2}+\theta .
$$

In particular Fix $\left\langle\alpha_{3}\right\rangle$ is a hyperelliptic curve of genus 3.

Proof. Since $\alpha_{3}$ fixes both $\theta$ and $\rho$, we have $\overline{\mathbb{F}}_{p}(\theta, \rho) \subseteq F i x\left\langle\alpha_{3}\right\rangle$. As the degree of the function field extension $\overline{\mathbb{F}}_{p}(x, y): \overline{\mathbb{F}}_{p}(\theta, \rho)$ is clearly 2, we have that $F i x\left\langle\alpha_{3}\right\rangle=\overline{\mathbb{F}}_{p}(\theta, \rho)$. By Equation (10), $\rho^{2}=y^{2} \theta=\left(x^{12}-a x^{10}-33 x^{8}+2 a x^{6}-33 x^{4}-a x^{2}+1\right) \theta=\theta^{7}-a \theta^{6}-33 \theta^{5}+2 a \theta^{4}-33 \theta^{3}-a \theta^{2}+\theta$. Consider the polynomial $f_{2}(X)=X^{7}-a X^{6}-33 X^{5}+2 a X^{4}-33 X^{3}-a X^{2}+X$. Then $f_{2}(X)$ has no multple roots over $\overline{\mathbb{F}}_{p}$ since the resultant of $f_{2}(X)$ and $f_{2}^{\prime}(X)$ is equal to $\left(a^{2}+108\right)^{4} \neq 0$. From [74, Proposition 3.7.3 and Corollary 3.7.4], Fix $\left\langle\alpha_{3}\right\rangle$ is a hyperelliptic function field of genus 3.

At this point, we get a first decomposition of the Jacobian of $\mathcal{C}_{a}$ as a straightforward application of Corollary 1.2 .

Corollary 3.5. Let $q=p^{h}$ where $h \in \mathbb{N}$ and $p$ is an odd prime. Let $a \in \mathbb{F}_{q^{2}}$ with $a^{2}+108 \neq 0$. Then the Jacobian variety $J_{a}$ of the curve $\mathcal{C}_{a}$ defined in (10) decomposes as

$$
J_{a} \sim J_{a, 2} \times J_{a, 3},
$$


where $J_{a, 2}$ is the Jacobian variety of the hyperelliptic curve of genus 2

$$
\mathcal{C}_{a, 2}: Y^{2}=X^{6}-a X^{5}-33 X^{4}+2 a X^{3}-33 X^{2}-a X+1,
$$

while $J_{a, 3}$ is the Jacobian variety of the hyperelliptic curve of genus 3

$$
\mathcal{C}_{a, 3}: Y^{2}=X^{7}-a X^{6}-33 X^{5}+2 a X^{4}-33 X^{3}-a X^{2}+X .
$$

Now, a decomposition of the Jacobian $J_{a, 2}$ of the curve $\mathcal{C}_{a, 2}$ whose function field is defined by Equation (11)), is obtained by applying Theorem 1.1 to the automorphism group $G_{2}=\left\langle\beta_{1}, \beta_{2}\right\rangle$ with

$$
\begin{gathered}
\beta_{1}:(\theta, \eta) \rightarrow(\theta,-\eta), \\
\beta_{2}:(\theta, \eta) \rightarrow\left(1 / \theta, \eta / \theta^{3}\right) .
\end{gathered}
$$

It is easily seen that $\beta_{1}, \beta_{2} \in \operatorname{Aut}\left(\mathcal{C}_{a, 2}\right)$ and that $G_{2}=\left\langle\beta_{1}, \beta_{2}\right\rangle$ is elementary abelian of order 4. Also, since $\beta_{1}$ is the hyperelliptic involution of $\mathcal{C}_{a, 2}$ we know that both $F i x\left\langle\beta_{1}\right\rangle=\overline{\mathbb{F}}_{p}(\theta)$ and Fix $G_{2}=\overline{\mathbb{F}}_{p}(\theta+1 / \theta)$ are rational. We will prove that $J_{a, 2}$ decomposes completely as a power of the Jacobian of a unique elliptic curve. First, we compute the fixed fields of $\left\langle\beta_{2}\right\rangle$ and $\left\langle\beta_{3}\right\rangle=\left\langle\beta_{1} \beta_{2}\right\rangle$.

Lemma 3.6. Let $q=p^{h}$ where $h \in \mathbb{N}$ and $p$ is an odd prime. Let $a \in \mathbb{F}_{q^{2}}$ with $a^{2}+108 \neq 0$. Then Fix $\left\langle\beta_{2}\right\rangle=\overline{\mathbb{F}}_{p}(\tilde{x}, \tilde{y})$ where

$$
\tilde{y}^{2}=4 \tilde{x}^{3}+(a+6) \tilde{x}^{2}+(a-6) \tilde{x}-4 .
$$

In particular Fix $\left\langle\beta_{2}\right\rangle$ is elliptic.

Proof. Let

$$
\tilde{x}=-\frac{(\theta-1)^{2}}{(\theta+1)^{2}} \quad \text { and } \quad \tilde{y}=-\frac{\eta}{(\theta+1)^{3}} .
$$

Note that both $\tilde{x}$ and $\tilde{y}$ are fixed by $\beta_{2}$; also, the field extension $\overline{\mathbb{F}}_{p}(\theta, \eta): \overline{\mathbb{F}}_{p}(\tilde{x}, \tilde{y})$ has degree 2 , whence $\operatorname{Fix}\left\langle\beta_{2}\right\rangle=\overline{\mathbb{F}}_{p}(\tilde{x}, \tilde{y})$. By Equation (11) $\tilde{y}^{2}=\tilde{x}^{3}+\frac{a+6}{4} \tilde{x}^{2}+\frac{a-6}{4} \tilde{x}-1$. The polynomial $X^{3}+\frac{a+6}{4} X^{2}+\frac{a-6}{4} X-1$ has no multiple roots since $\left(a^{2}+108\right) \neq 0$ and hence $F i x\left\langle\beta_{2}\right\rangle$ is elliptic. The claim follows considering the isomorphism $(\tilde{x}, \tilde{y}) \mapsto(\tilde{x}, \tilde{y} / 2)$.

Lemma 3.7. Let $q=p^{h}$ where $h \in \mathbb{N}$ and $p$ is an odd prime. Let $a \in \mathbb{F}_{q^{2}}$ with $a^{2}+108 \neq 0$. Let $\beta_{3}=\beta_{1} \beta_{2}$ so that $\beta_{3}:(\theta, \eta) \mapsto\left(1 / \theta,-\eta / \theta^{3}\right)$. Then Fix $\left\langle\beta_{3}\right\rangle=\overline{\mathbb{F}}_{p}(\bar{x}, \bar{y})$ where

$$
\bar{y}^{2}=4 \bar{x}^{3}+(-a+6) \bar{x}^{2}+(-a-6) \bar{x}-4 .
$$

In particular Fix $\left\langle\beta_{3}\right\rangle$ is elliptic.

Proof. Let

$$
\bar{x}=-\frac{(\theta+1)^{2}}{(\theta-1)^{2}} \quad \text { and } \quad \bar{y}=\frac{\eta}{(\theta-1)^{3}} .
$$

Then the proof is analogous to that of Lemma 3.6. 
Remark 3.8. Let $q=p^{h}$ where $h \in \mathbb{N}$ and $p$ is an odd prime. Let $a \in \mathbb{F}_{q^{2}}$ with $a^{2}+108 \neq 0$. Then the quotient curves $\mathcal{C}_{a, 2} /\left\langle\beta_{2}\right\rangle$ and $\mathcal{C}_{a, 2} /\left\langle\beta_{3}\right\rangle$ are $\mathbb{F}_{q^{2}}$-isomorphic. The isomorphism between the two curves is given by

$$
\varphi:(\tilde{x}, \tilde{y}) \mapsto(-\tilde{x}-1, \xi \tilde{y})
$$

where $\xi^{2}=-1$.

At this point the complete decomposition of $J_{a, 2}$ follows by applying Corollary 1.2 to $G_{2}$.

Corollary 3.9. Let $q=p^{h}$ where $h \in \mathbb{N}$ and $p$ is an odd prime. Let $a \in \mathbb{F}_{q^{2}}$ with $a^{2}+108 \neq 0$. Then the Jacobian variety $J_{a, 2}$ of the curve $\mathcal{C}_{a, 2}$ defined in (14) decomposes as $J_{a, 2} \sim J_{E_{1}}^{2}$ where $J_{E_{1}}$ is the Jacobian variety of the elliptic curve

$$
E_{1}: Y^{2}=4 X^{3}+(a+6) X^{2}+(a-6) X-4 .
$$

Furthermore, the Jacobian variety $J_{a}$ of the curve $\mathcal{C}_{a}$ defined in (10) decomposes as

$$
J_{a} \sim J_{E_{1}}^{2} \times J_{a, 3}
$$

where $J_{a, 3}$ is as in Corollary 3.5.

We proceed now with the complete decomposition of the Jacobian $J_{a, 3}$ of the curve $\mathcal{C}_{a, 3}$, whose function field $\overline{\mathbb{F}}_{p}(\rho, \theta)$ is defined in Equation (12), by applying Theorem 1.1, with respect to the automorphism group $G_{3}=\left\langle\gamma_{1}, \gamma_{2}\right\rangle$, where

$$
\begin{gathered}
\gamma_{1}:(\theta, \rho) \rightarrow(\theta,-\rho), \\
\gamma_{2}:(\theta, \rho) \rightarrow\left(1 / \theta, \rho / \theta^{4}\right) .
\end{gathered}
$$

It is easily seen that $\gamma_{1}, \gamma_{2} \in \operatorname{Aut}\left(\mathcal{C}_{a, 3}\right)$ and that $G_{3}=\left\langle\gamma_{1}, \gamma_{2}\right\rangle$ is elementary abelian of order 4 . Since $\gamma_{1}$ is the hyperelliptic involution of $\mathcal{C}_{a, c}$, both $F i x\left\langle\gamma_{1}\right\rangle=\overline{\mathbb{F}}_{p}(\theta)$ and $F i x G_{3}$ are rational. We first show that $J_{a, 3}$ decomposes as a the product of the Jacobians of an elliptic curve and of a curve of genus 2 .

Lemma 3.10. Let $q=p^{h}$ where $h \in \mathbb{N}$ and $p$ is an odd prime. Let $a \in \mathbb{F}_{q^{2}}$ with $a^{2}+108 \neq 0$. Then Fix $\left\langle\gamma_{2}\right\rangle=\overline{\mathbb{F}}_{p}(\delta, \nu)$ where

$$
\nu^{2}=\delta^{3}-a \delta^{2}-36 \delta+4 a
$$

In particular Fix $\left\langle\gamma_{2}\right\rangle$ is elliptic.

Proof. Let $\delta=\left(\theta^{2}+1\right) / \theta=\theta+1 / \theta=\theta+\gamma_{2}(\theta)$ and $\nu=-\rho / \theta^{2}$. Then $\delta$ and $\rho$ are fixed by $\gamma_{2}$ since $\gamma_{2}(\delta)=\gamma_{2}(\theta)+\gamma_{2}^{2}(\theta)=\gamma_{2}(\theta)+\theta$ and $\gamma_{2}(\nu)=\left(-\rho / \theta^{4}\right) \cdot \theta^{2}=-\rho / \theta^{2}=\nu$. Also from (12),

$$
\begin{aligned}
\nu^{2} & =\frac{\rho^{2}}{\theta^{4}}=\frac{\theta^{7}-a \theta^{6}-33 \theta^{5}+2 a \theta^{4}-33 \theta^{3}-a \theta^{2}+\theta}{\theta^{4}} \\
& =\frac{\theta^{6}-a \theta^{5}-33 \theta^{4}+2 a \theta^{3}-33 \theta^{2}-a \theta+1}{\theta^{3}},
\end{aligned}
$$

whereas

$$
\begin{aligned}
\delta^{3}-a \delta^{2}-36 \delta+4 a & =\frac{\left(\theta^{2}+1\right)^{3}-a \theta\left(\theta^{2}+1\right)^{2}-36 \theta^{2}\left(\theta^{2}+1\right)+4 a \theta^{3}}{\theta^{3}} \\
& =\frac{\theta^{6}-a \theta^{5}-33 \theta^{4}+2 a \theta^{3}-33 \theta^{2}-a \theta+1}{\theta^{3}} .
\end{aligned}
$$


Then the rest of the proof is analogous to that of Lemma 3.6.

In the following remark we show that the quotient curve $\mathcal{C}_{a, 3} /\left\langle\gamma_{2}\right\rangle$ and $E_{1}$ are $\mathbb{F}_{q^{2}}$-isomorphic.

Remark 3.11. Let $q=p^{h}$ where $h \in \mathbb{N}$ and $p$ is an odd prime. Let $a \in \mathbb{F}_{q^{2}}$ with $a^{2}+108 \neq 0$. Then the elliptic curves $E_{1}: Y^{2}=4 X^{3}+(a+6) X^{2}+(a-6) X-4$ and $\mathcal{C}_{a, 3} /\left\langle\gamma_{2}\right\rangle: Y^{2}=X^{3}-a X^{2}-36 X+4 a$ are $\mathbb{F}_{q^{2}}$-isomorphic through the birational morphism $\varphi: E_{1} \rightarrow \mathcal{C}_{a, 3} /\left\langle\gamma_{2}\right\rangle$ given by

$$
\varphi(X, Y) \mapsto(-4 X-2,4 \xi Y)
$$

where $\xi^{2}=-1$.

Lemma 3.12. Let $q=p^{h}$ where $h \in \mathbb{N}$ and $p$ is an odd prime. Let $a \in \mathbb{F}_{q^{2}}$ with $a^{2}+108 \neq 0$. Let $\gamma_{3}=\gamma_{1} \gamma_{2}$ so that $\gamma_{3}:(\theta, \rho) \mapsto\left(1 / \theta,-\rho / \theta^{4}\right)$. Then Fix $\left\langle\gamma_{3}\right\rangle=\overline{\mathbb{F}}_{p}(\delta, \epsilon)$ where

$$
\epsilon^{2}=\delta^{5}-a \delta^{4}-40 \delta^{3}+8 a \delta^{2}+144 \delta-16 a=\left(\delta^{2}-4\right)\left(\delta^{3}-a \delta^{2}-36 \delta+4 a\right) .
$$

In particular, Fix $\left\langle\gamma_{3}\right\rangle$ is hyperelliptic of genus 2 .

Proof. Let $\delta=\left(\theta^{2}+1\right) / \theta=\theta+\theta=\theta+\gamma_{3}(\theta)$ and $\epsilon=\left(\theta^{2} \rho-\rho\right) / \theta^{3}$. Then $\delta$ and $\epsilon$ are fixed by $\gamma_{3}$ since $\gamma_{3}(\delta)=\gamma_{3}(\theta)+\gamma_{3}^{2}(\theta)=\gamma_{3}(\theta)+\theta$ and $\gamma_{3}(\epsilon)=\left(-\rho / \theta^{6}+\rho / \theta^{4}\right) \cdot \theta^{3}=\left(-\rho+\rho \theta^{2}\right) / \theta^{3}=\epsilon$. Using Equation (12) one can easily check that $\epsilon^{2}=\delta^{5}-a \delta^{4}-40 \delta^{3}+8 a \delta^{2}+144 \delta-16 a$ holds. Arguing as in the previous proofs, we have that $\overline{\mathbb{F}}_{p}(\delta, \epsilon)$ coincides with $\operatorname{Fix}\left\langle\gamma_{3}\right\rangle$ and it is hyperelliptic of genus 2 .

The following corollary summarizes the results obtained in this section so far.

Corollary 3.13. Let $q=p^{h}$ where $h \in \mathbb{N}$ and $p$ is an odd prime. Let $a \in \mathbb{F}_{q^{2}}$ with $a^{2}+108 \neq 0$. Then the Jacobian variety $J_{a, 3}$ of the curve $\mathcal{C}_{a, 3}$ defined in (15) decomposes as $J_{a, 3} \sim J_{a, 3,2} \times J_{E_{1}}$ where $J_{E_{1}}$ is the Jacobian variety of the elliptic curve

$$
E_{1}: Y^{2}=4 X^{3}+(a+6) X^{2}+(a-6) X-4,
$$

and $J_{a, 3,2}$ is the Jacobian variety of the genus 2 curve

$$
\mathcal{C}_{a, 3,2}: Y^{2}=X^{5}-a X^{4}-40 X^{3}+8 a X^{2}+144 X-16 a .
$$

Furthermore, the Jacobian variety $J_{a}$ of the curve $\mathcal{C}_{a}$ defined in (10) decomposes as

$$
J_{a} \sim J_{E_{1}}^{3} \times J_{a, 3,2}
$$

In the rest of the section we investigate the curve $\mathcal{C}_{a, 3,2}$, whose function field is $\overline{\mathbb{F}}_{q}(\delta, \epsilon)$, where Equation (21) holds.

For $k \in \mathbb{F}_{p^{2}}, k \neq \pm 2$ and $k \neq \pm 6$, let $\lambda=\frac{12-k^{2}}{2 k}$ and $a=\frac{\lambda^{3}-36 \lambda}{\lambda^{2}-4}$. Then, by straightforward computation

$$
\epsilon^{2}=\left(\delta^{2}-4\right)(\delta-\lambda)\left(\delta+\frac{2 \lambda+12}{\lambda-2}\right)\left(\delta+\frac{-2 \lambda+12}{\lambda+2}\right) .
$$

We consider the group generated by the hyperelliptic involution and

$$
\psi_{i}(\delta, \epsilon)=\left(\frac{\lambda \delta+12}{\delta-\lambda}, \frac{(-1)^{i} \mu \epsilon}{(\delta-\lambda)^{3}}\right),
$$


$i=0,1$, where $\mu^{2}=\left(\lambda^{2}+12\right)^{3}$, which is elementary abelian of order 4 . Note that $\mu \in \mathbb{F}_{p^{2}}$, since $\lambda^{2}+12=\left(\frac{k^{2}+12}{2 k}\right)^{2}$ is a square in $\mathbb{F}_{p^{2}}$.

The functions

$$
\frac{(\delta-\lambda)\left(\lambda^{2}+12\right)+(-1)^{i} \mu}{(\delta-\lambda)^{2}\left(\lambda^{2}+12\right)} \cdot \epsilon, \quad \frac{\delta^{2}+12}{\delta-\lambda}
$$

are fixed by $\psi_{i}$.

By direct computations, the quotient curves $\mathcal{C}_{a, 3,2}^{i}=\mathcal{C}_{a, 3,2} /\left\langle\psi_{i}\right\rangle$ have equations

$$
\begin{aligned}
\left(\frac{1}{4} k^{4}-10 k^{2}+36\right) \epsilon^{2}= & \left(\frac{1}{4} k^{4}-10 k^{2}+36\right) \delta^{3}+\left(\frac{1}{2} k^{5}-36 k^{3}+264 k\right) \delta^{2} \\
& +\left(-32 k^{4}+640 k^{2}\right) \delta+512 k^{3} ; \\
\left(\frac{1}{4} k^{5}-10 k^{3}+36 k\right) \epsilon^{2}= & \left(\frac{1}{4} k^{5}-10 k^{3}+36 k\right) \delta^{3}+\left(-22 k^{4}+432 k^{2}-864\right) \delta^{2} \\
& +\left(640 k^{3}-4608 k\right) \delta-6144 k^{2} .
\end{aligned}
$$

It turns out that the two elliptic curves are isogenous over $\mathbb{F}_{p^{2}}$ via the isogeny $\varphi: \mathcal{C}_{a, 3,2}^{0} \rightarrow \mathcal{C}_{a, 3,2}^{1}$ with

$$
\varphi:(\delta, \epsilon) \mapsto\left(\frac{a_{3} \delta^{3}+a_{2} \delta^{2}+a_{1} \delta+a_{0}}{(\delta+c)^{2}}, \frac{\delta \epsilon\left(b_{2} \delta^{2}+b_{1} \delta+b_{0}\right)}{(\delta+c)^{3}}\right)
$$

where

$$
\begin{array}{r}
a_{0}=-\frac{1536 k}{\left.\left(k^{2}-36\right)\right)^{2} k}, \quad a_{1}=-\frac{3072 k}{\left(k^{2}-36\right) k}, \quad a_{2}=\frac{32}{k}, \quad a_{3}=\frac{4}{k^{2}} \\
b_{0}=-\frac{2048}{k^{3}-36 k}, \quad b_{1}=\frac{1536 k^{2}}{k^{2}-36}, \quad b_{2}=\frac{8}{k^{3}}, \quad c=-\frac{64 k}{k^{2}-36} .
\end{array}
$$

Now we need to investigate whether $\mathcal{C}_{a, 3,2}^{0}$ and $E_{1}$ are isogenous or not. To this end, we will consider the theory of modular polynomials, for which we refer to [12] and the web database [13]. We proceed by arguing as in [72, Section 1].

Let $\Phi_{\ell} \in \mathbb{Z}[X, Y]$ be the classical modular polynomial of degree $\ell$. It parametrizes pairs of $\ell$ isogenous elliptic curves in terms of their $j$-invariants. More precisely, over a field $\mathbb{F}$ of characteristic not equal to $\ell$, the modular equation $\Phi_{\ell}\left(j_{1}, j_{2}\right)=0$ holds if and only if $j_{1}$ and $j_{2}$ are the $j$-invariants of elliptic curves defined over $\mathbb{F}$ that are related by a cyclic isogeny of degree $\ell$.

Here, $\mathbb{F}$ is a finite field $\mathbb{F}_{p^{2}}$. Let $\bar{j}(k)$, and $\widetilde{j}(k)$ be the $j$-invariants of $\mathcal{C}_{a, 3,2}^{0}$ and $E_{1}$, respectively, both expressed as a function of $k$. We have

$$
\bar{j}(k)=\frac{\left(k^{2}+12\right)^{3}\left(k^{6}-60 k^{4}+1200 k^{2}+192\right)^{3}}{64\left(k^{2}-36\right)^{2}\left(k^{2}-4\right)^{6} k^{2}}, \quad \widetilde{j}(k)=\frac{4\left(k^{2}+12\right)^{6}}{\left(k^{2}-36\right)^{2}\left(k^{2}-4\right)^{2} k^{2}} .
$$


We consider the case $\ell=3$. The class of elliptic curves 3 -isogenous to $E_{1}$ can be determined by computing (if any) the roots of $\Phi_{3}(Y)=\Phi_{3}\left(j\left(E_{1}\right), Y\right) \in \mathbb{F}_{p^{2}}[Y]$ over $\mathbb{F}_{p^{2}}$. Hence we search for $\bar{k} \in \mathbb{F}_{p^{2}}$ with $\Phi_{3}(\bar{j}(\bar{k}), \widetilde{j}(\bar{k}))=0$ satisfying Condition (9) .

We are not able a priori to decide whether the 3 -isogeny is between $E_{1}$ and $C_{a, 3,2}^{0}$ or its twists. So, the condition $\Phi_{3}(\bar{j}(\bar{k}), \widetilde{j}(\bar{k}))=0$ ensures the existence of a 3-isogeny over $\mathbb{F}_{p^{4}}$ between $\mathcal{C}_{a, 3,2}^{0}$ and $E_{1}$. Thus we look for explicit descriptions of such 3 -isogenies and check if they are defined over $\mathbb{F}_{p^{2}}$.

- $\bar{k}^{2}=36 / 5$. In this case the 3-isogeny between $\mathcal{C}_{a, 3,2}^{0}$ and $E_{1}$ is $(x, y) \mapsto\left(\frac{\psi_{x}}{\theta_{x}}, \frac{\psi_{y}}{\theta_{y}}\right)$, where

$$
\begin{aligned}
\psi_{x}= & -360 x^{3} \bar{k}+2160 x^{3}+14688 x^{2} \bar{k}-36288 x^{2}-138240 x \bar{k}+331776 x+368640 \bar{k}-1105920 ; \\
\theta_{x}= & 360 x^{3} \bar{k}-1296 x^{3}-10368 x^{2}-69120 x \bar{k}+82944 x+92160 \bar{k}-552960 ; \\
\psi_{y}= & y\left(103680 x^{4} \bar{k}-124416 x^{4}-1105920 x^{3} \bar{k}+6635520 x^{3}+17694720 x^{2} y \bar{k}\right. \\
& \left.-21233664 x^{2}-17694720 x \bar{k}+106168320 x\right) ; \\
\theta_{y}= & -6480 x^{6} \bar{k}+12960 x^{6}-103680 x^{5} \bar{k}-103680 x^{4} \bar{k}-6842880 x^{4}-15482880 x^{3} \bar{k} \\
& -53084160 x^{3}-225607680 x^{2} \bar{k}-79626240 x^{2}-2548039680 x-1415577600 \bar{k} .
\end{aligned}
$$

- $\bar{k}^{2}=-4 / 7$. In this case the 3-isogeny between $\mathcal{C}_{a, 3,2}^{0}$ and $E_{1}$ is $(x, y) \mapsto\left(\frac{\psi_{x}}{\theta_{x}}, \frac{\psi_{y}}{\theta_{y}}\right)$, where

$$
\begin{aligned}
\psi_{x}= & 9756997844651904 x^{3} \bar{k}+1773999608118528 x^{3}-236713557425574852 x^{2} \bar{k} \\
& -88881615135103896 x^{2}-149484312145104132 x \bar{k}+93592177008289512 x \\
& +108081789310424734 \bar{k}+80629249554918068 ; \\
\theta_{x}= & -9756997844651904 x^{3} \bar{k}+14445425380393728 x^{3}+117767323565720640 x^{2} \bar{k} \\
& +35210207348410752 x^{2}+76832921522684208 x \bar{k}-181553980415546592 x \\
& -164874109067025579 \bar{k}-34657310309390402 ; \\
\psi_{y}= & 5 y\left(3457479429497341093236434568000000 x^{4} \bar{k}-1014193965985886720682687473280000 x^{4}\right. \\
& -15545163634077077821994778380160000 x^{3} \bar{k}-2826393388014014149453596069120000 x^{3} \\
& -117406724988018917190005308164803520 x^{2} \bar{k}+35214070050544757907472923900067776 x^{2} \\
& +897502873974695889338633745055844520 x \bar{k}+43112385947023817822534160466259664 x \\
& -86384192632333382597513730705421100 \bar{k}-65824077521259664661193935638615080) / 6 ; \\
= & 12012271389339333626787155527680000 x^{6} \bar{k}+4636315273078339294549428449280000 x^{6} \\
& +58558963160486989619604042762240000 x^{5} \bar{k}-111920992422190876567021530009600000 x^{5} \\
& -753377091175625014779032837560320000 x^{4} \bar{k}-171585897605402253148919969648640000 x^{4} \\
& -455341963965646265965574769587040000 x^{3} \bar{k}+1538861060386352212314316012831680000 x^{3} \\
& +3093062942957171752624793406954480000 x^{2} \bar{k}+374348928145257960497936027483040000 x^{2} \\
& +274459963096197145160822714714460000 x \bar{k}-1900058781725511243895076291080920000 x \\
& -855848892852162349927591124235841875 \bar{k}-45599538718475703951502910235251250 .
\end{aligned}
$$


- $\bar{k}^{2}=24 \bar{k}+36$. In this case the 3-isogeny between $\mathcal{C}_{a, 32}^{0}$ and $E_{1}$ is $(x, y) \mapsto\left(A(x), c y A^{\prime}(x)\right)$, $A(x)=\frac{a_{3} x^{3}+a_{2} x^{2}+a_{1} x+a_{0}}{x^{3}+b_{2} x^{2}+b_{1} x+b_{0}}, c^{2}-1 / 3 c-1 / 9=0$, and

$$
\begin{aligned}
\bar{k} a_{3}+2 / 7 \bar{k}+6 / 7 a_{3} & =0 \\
a_{2}^{2}+276 / 5 a_{2}+17424 / 25 & =0 \\
a_{1}^{2}-384 a_{1}+36864 / 5 & =0 \\
a_{0}^{2}+512 a_{0}+65536 / 5 & =0 \\
b_{2}^{2}-12 b_{2}-1584 / 5 & =0 \\
b_{1}^{2}-192 b_{1}+36864 / 5 & =0 \\
b_{0}^{2}+256 b_{0}+65536 / 5 & =0 .
\end{aligned}
$$

- $\bar{k}^{2}=-24 \bar{k}+36$. In this case the 3-isogeny between $\mathcal{C}_{a, 32}^{0}$ and $E_{1}$ is $(x, y) \mapsto\left(A(x), c y A^{\prime}(x)\right)$, $A(x)=\frac{a_{3} x^{3}+a_{2} x^{2}+a_{1} x+a_{0}}{x^{3}+b_{2} x^{2}}, c^{2}-1 / 3 c-1 / 9=0$, and

$$
\begin{aligned}
\bar{k} a_{3}-2 / 5 \bar{k}-6 / 5 a_{3} & =0 \\
a_{2}^{2}-132 a_{2}+11376 / 5 & =0 \\
a_{1}^{2}-768 a_{1}+589824 / 5 & =0 \\
a_{0}^{2}-1024 a_{0}+1048576 / 5 & =0 \\
b_{2}^{2}+48 b_{2}-144 & =0 .
\end{aligned}
$$

We summarize all the results about the curve $\mathcal{C}_{a}$ in the following theorem.

Theorem 3.14. Let $q=p^{h}$, where $h \in \mathbb{N}$ and $p$ is an odd prime. Let

$$
a=\frac{-k^{6}+180 k^{4}-2160 k^{2}+1728}{2 k^{5}-80 k^{3}+288 k} \in \mathbb{F}_{q^{2}}, \quad k \in \mathbb{F}_{q^{2}}, \quad a^{2}+108 \neq 0 .
$$

If $k^{2}=36 / 5, k^{2}=-4 / 7$, or $k^{2}= \pm 24 k^{2}+36$, then $a \in \mathbb{F}_{p^{2}}$ and the Jacobian variety $\mathcal{J}_{a}$ of the curve $\mathcal{C}_{a}$ defined in (10) is $\mathbb{F}_{p^{2}}$-isogenous to the fifth power of the Jacobian variety $J_{E_{1}}$ of the elliptic curve

$$
E_{1}: Y^{2}=4 X^{3}+(a+6) X^{2}+(a-6) X-4 .
$$

In particular, there exist infinitely many primes $p$ such that $\mathcal{C}_{a}$ is either maximal or minimal over $\mathbb{F}_{p^{2}}$.

Proof. We only need to prove that there exists a fixed elliptic curve $E$ defined over $\mathbb{Q}$ such that $E_{1}$ and $E$ are $\mathbb{F}_{p^{4}}$-isogenous for any $p$. This is clear since the $j$-invariant of $E_{1}$ is a rational number.

Using the software MAGMA [9] we determined all the primes $p$ less than 100000 such that one of the above conditions on $\bar{k} \in \mathbb{F}_{p}$ is satisfied and $E_{1}$ is $\mathbb{F}_{p^{2} \text {-maximal. }}$

Theorem 3.15. For $a$ and $p$ as in Tables 1, 2, 3, 4, the curve $\mathcal{C}_{a}$ defined in (15) is $\mathbb{F}_{p^{2}}$-maximal. 


\begin{tabular}{|l|l||l|l||l|l||l|l||l|l|}
\hline$p$ & $a$ & $p$ & $a$ & $p$ & $a$ & $p$ & $a$ & $p$ & $a$ \\
\hline 11 & 0 & 131 & 75 & 251 & 221 & 491 & 254 & 599 & 24 \\
1439 & 984 & 3371 & 933 & 5639 & 4445 & 5879 & 1294 & 6971 & 2129 \\
7079 & 6366 & 8039 & 4751 & 8291 & 5367 & 9839 & 7465 & 10799 & 1835 \\
11171 & 5951 & 12119 & 4310 & 14879 & 2439 & 16931 & 14808 & 17159 & 4688 \\
18839 & 17890 & 23039 & 5286 & 23159 & 16126 & 25919 & 24335 & 50291 & 5355 \\
53411 & 49474 & 53639 & 10108 & 59051 & 25275 & 69371 & 51864 & 74771 & 11482 \\
74891 & 48023 & 75239 & 47655 & 81119 & 7710 & 81359 & 48587 & & \\
\hline
\end{tabular}

TABle 1. Values $a \in \mathbb{F}_{p}$ for which $\mathcal{C}_{a, 3}$ defined in (15) is $\mathbb{F}_{p^{2}}$-maximal $\left(\bar{k}^{2}=36 / 5\right)$

\begin{tabular}{|l|l||l|l||l|l||l|l||l|l|}
\hline$p$ & $a$ & $p$ & $a$ & $p$ & $a$ & $p$ & $a$ & $p$ & $a$ \\
\hline 11 & 0 & 23 & 0 & 71 & 45 & 263 & 185 & 1031 & 881 \\
1283 & 1276 & 1583 & 207 & 2039 & 1586 & 2087 & 752 & 2543 & 565 \\
2843 & 2158 & 7823 & 2710 & 9851 & 6895 & 10859 & 7298 & 12107 & 1665 \\
15647 & 4803 & 18719 & 9865 & 23459 & 17387 & 25463 & 14115 & 26723 & 22923 \\
27791 & 27198 & 29759 & 25238 & 31511 & 30573 & 32579 & 25335 & 33791 & 31173 \\
34283 & 3491 & 35099 & 20799 & 37619 & 25640 & 54959 & 54405 & 56519 & 47622 \\
61007 & 47142 & 61559 & 42225 & 67043 & 32831 & 71339 & 1126 & 71843 & 10626 \\
89783 & 68842 & 95027 & 9806 & & & & & & \\
\hline
\end{tabular}

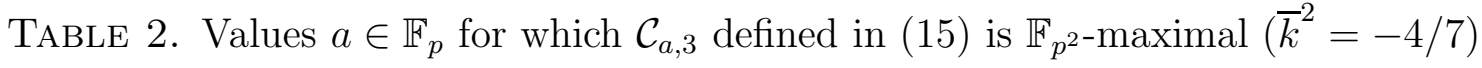

\begin{tabular}{|l|l||l|l||l|l||l|l||l|l|}
\hline$p$ & $a$ & $p$ & $a$ & $p$ & $a$ & $p$ & $a$ & $p$ & $a$ \\
\hline 11 & 0 & 131 & 56 & 251 & 221 & 491 & 237 & 599 & 575 \\
1439 & 455 & 3371 & 2438 & 5639 & 1194 & 5879 & 1294 & 6971 & 2129 \\
7079 & 713 & 8039 & 3288 & 8291 & 2924 & 9839 & 7465 & 10799 & 1835 \\
11171 & 5951 & 12119 & 4310 & 14879 & 12440 & 16931 & 2123 & 17159 & 12471 \\
18839 & 17890 & 23039 & 5286 & 23159 & 7033 & 25919 & 24335 & 50291 & 5355 \\
53411 & 3937 & 53639 & 10108 & 59051 & 33776 & 69371 & 51864 & 74771 & 63289 \\
74891 & 48023 & 75239 & 27584 & 81119 & 7710 & 81359 & 48587 & & \\
\hline
\end{tabular}

TABLE 3. Values $a \in \mathbb{F}_{p}$ for which $\mathcal{C}_{a, 3}$ defined in (15) is $\mathbb{F}_{p^{2}-\text { maximal }}\left(\bar{k}^{2}=24 \bar{k}+36\right)$

\section{A characterization in terms of automorphism groups}

In this section we extend to the positive characteristic case a characterization of curves with equation (10) in terms of their automorphism group, provided by Shaska in [70].

Let $q=p^{h}$ where $h \in \mathbb{N}$ and $p$ is an odd prime. Let $a \in \mathbb{F}_{q^{2}}$ with $a^{2}+108 \neq 0$. Denote by $\overline{\mathbb{F}}_{p}$ the algebraic closure of $\mathbb{F}_{q^{2}}$. We consider the family of algebraic curves $\mathcal{C}_{a}$ as described in (10). 


\begin{tabular}{|l|l||l|l||l|l||l|l||l|l|}
\hline$p$ & $a$ & $p$ & $a$ & $p$ & $a$ & $p$ & $a$ & $p$ & $a$ \\
\hline 11 & 0 & 131 & 56 & 251 & 30 & 491 & 237 & 599 & 24 \\
1439 & 455 & 3371 & 933 & 5639 & 4445 & 5879 & 4585 & 6971 & 4842 \\
7079 & 6366 & 8039 & 3288 & 8291 & 2924 & 9839 & 2374 & 10799 & 1835 \\
11171 & 5220 & 12119 & 4310 & 14879 & 2439 & 16931 & 14808 & 17159 & 12471 \\
18839 & 17890 & 23039 & 5286 & 23159 & 16126 & 25919 & 1584 & 50291 & 5355 \\
53411 & 3937 & 53639 & 10108 & 59051 & 33776 & 69371 & 17507 & 74771 & 11482 \\
74891 & 48023 & 75239 & 27584 & 81119 & 73409 & 81359 & 32772 & & \\
\hline
\end{tabular}

TABLE 4. Values $a \in \mathbb{F}_{p}$ for which $\mathcal{C}_{a, 3}$ defined in (15) is $\mathbb{F}_{p^{2}}$-maximal $\left(\bar{k}^{2}=-24 \bar{k}+36\right)$

We know that $g\left(\mathcal{C}_{a}\right)=5, \mathcal{C}_{a}$ is hyperelliptic and that the hyperelliptic involution is

$$
\alpha_{1}:(X, Y) \mapsto(X,-Y) .
$$

Our first aim is to show that $\operatorname{Aut}\left(\mathcal{C}_{a}\right)$ admits a subgroup $H$ with $H \cong \mathrm{A}_{4} \times \mathrm{C}_{2} \cong \operatorname{SmallGroup}(24,13)$ where $\mathrm{A}_{4}$ denotes the alternating group in 4 letters. Then we prove that if $\mathcal{X}_{5}$ is an hyperelliptic curve of genus 5 admitting an automorphism group isomorphic to $H$ then $\mathcal{X}$ is $\overline{\mathbb{F}}_{p}$-birationally equivalent to a curve $\mathcal{C}_{a}$ in (10).

Let $i \in \mathbb{F}_{q^{2}}$ with $i^{2}=-1$. Consider the following map

$$
\beta:(X, Y) \mapsto\left(\frac{X-i}{X+i}, \frac{8 i Y}{(X+i)^{6}}\right) .
$$

It is easily seen that $\beta \in \operatorname{Aut}\left(\mathcal{C}_{a}\right)$. Also, $\beta$ has order 3 . Involutory automorphisms of $\mathcal{C}_{a}$ are described as follows:

$$
\alpha_{2}:(X, Y) \mapsto(-X, Y) \quad \text { and } \quad \alpha_{3}:(X, Y) \mapsto\left(\frac{1}{X}, \frac{Y}{X^{6}}\right)
$$

The group generated by $\alpha_{1}, \alpha_{2}$, and $\alpha_{3}$ is an elementary abelian group of order 8 .

To show that $H_{1}=\left\langle\alpha_{2}, \beta\right\rangle \cong \mathrm{A}_{4}$ and that $\alpha_{1} \notin H_{1}$, we first observe that

$$
\beta^{-1} \alpha_{2} \beta=\alpha_{3} \text {. }
$$

Then $\beta$ normalizes the elementary abelian group of order 4 generated by $\alpha_{2}$ and $\alpha_{3}$. This implies that $H_{1} \cong\left(C_{2} \times C_{2}\right) \rtimes C_{3} \cong \mathrm{A}_{4}$. Also, $\alpha_{1} \notin\left\langle\alpha_{2}, \alpha_{3}\right\rangle$ yields $\alpha_{1} \notin H_{1}$. This proves that $\operatorname{Aut}\left(\mathcal{C}_{a}\right)$ contains a subgroup $H=\left\langle H_{1}, \alpha_{1}\right\rangle=H_{1} \times\left\langle\alpha_{1}\right\rangle \cong \mathrm{A}_{4} \times \mathrm{C}_{2} \cong \operatorname{SmallGroup}(24,13)$.

Assume now that, for an odd prime $p, \mathcal{X}_{5}$ is a hyperelliptic curve of genus 5 defined over a finite field of characteristic $p$ admitting an automorphism group $H \cong \mathrm{A}_{4} \times \mathrm{C}_{2}$. Our aim is to prove that $\mathcal{X}_{5}$ is $\overline{\mathbb{F}}_{p}$-birationally equivalent to a curve belonging to the family in (10).

The curve $\mathcal{X}_{5}$ admits an affine model

$$
\mathcal{X}_{5}: Y^{2}=F(X)=\prod_{i=1}^{12}\left(X-\gamma_{i}\right)
$$

where the points $P_{i}=\left(\gamma_{i}, 0\right), i=1, \ldots, 12$, are the Weierstrass points of $\mathcal{X}_{5}$; see [74, Proposition 6.2.3] and [46, Theorem 11.98]. Let $\overline{\mathbb{F}}_{p}(x, y)$ be the function field of $\mathcal{X}_{5}$. The hyperelliptic involution 
$\alpha_{1} \in H$ is $\alpha_{1}:(X, Y) \mapsto(X,-Y)$ and its fixed field is $\overline{\mathbb{F}}_{p}(x)$. The fixed points of $\alpha_{1}$ on $\mathcal{X}_{5}$ are exactly $P_{i}, i=1, \ldots, 12$.

Let $\bar{H}=H /\left\langle\alpha_{1}\right\rangle \cong \mathrm{A}_{4} \leq \operatorname{PGL}\left(2, \overline{\mathbb{F}}_{\mathrm{p}}\right)$. From [84, Theorem 1] (see also [46, Theorem A.8]), any subgroup of $\operatorname{PGL}\left(2, \overline{\mathbb{F}}_{p}\right)$ which is isomorphic to $\mathrm{A}_{4}$ is conjugated to $\bar{H}$ in $\operatorname{PGL}\left(2, \overline{\mathbb{F}}_{p}\right)$. This means that up to conjugation we can assume that $\bar{H}$, seen as an automorphism group of the rational function field $\overline{\mathbb{F}}_{p}(x)$, is generated by the following maps

$$
\theta_{1}: x \mapsto-x, \quad \text { and } \quad \theta_{2}: x \mapsto \frac{x-i}{x+i}
$$

where $i^{2}=-1$.

Lemma 4.1. The subgroup $H_{1}$ of $H$ with $H_{1} \cong \mathrm{A}_{4}$ acts sharply transitively on the set of Weierstrass points of $\mathcal{X}_{5}$.

Proof. Since $H_{1}$ is an automorphism group of $\mathcal{X}_{5}$, it acts on the set $W$ of its Weierstrass points. Since the stabilizer in $\operatorname{Aut}\left(\mathcal{X}_{5}\right)$ of a point $P \in W$ is cyclic from [46, Lemma 11.44] and clearly contains $\alpha_{1}$ we deduce that the 3 involutions in $H_{1}$ fix no points on $W$. Suppose that $H_{1}$ does not act transitively on $W$. Then the stabilizer $H_{1, P}$ of $P$ has order 3. A nontrivial element $\delta \in H_{1, P}$ fixes at least three points of $W$, since the number of fixed points must be congruent to 0 modulo 3 . Consider the element $\bar{\delta}$ induced by $\delta$ on $A u t\left(\mathcal{X}_{5} /\left\langle\alpha_{1}\right\rangle\right)$. Each point $\bar{P}$ lying under a fixed point of $\delta$ in $W$ is fixed by $\bar{\delta}$ On the other hand, by [46, Theorem $11.14(\mathrm{~d})], \bar{\delta}$ fixes exactly 2 points on $\mathcal{X}_{5} /\left\langle\alpha_{1}\right\rangle$, a contradiction.

Theorem 4.2. Let $p$ be an odd prime and let $\overline{\mathbb{F}}_{p}$ be the algebraic closure of $\mathbb{F}_{p}$. Let $\mathcal{X}_{5}: Y^{2}=F(X)$ be an hyperelliptic curve of genus 5 defined over $\overline{\mathbb{F}}_{p}$ and let $\alpha_{1}$ denote its hyperelliptic involution. If $\mathcal{X}_{5}$ admits an automorphism group $H \cong \mathrm{A}_{4} \times \mathrm{C}_{2}$ with $\bar{H}=H /\left\langle\alpha_{1}\right\rangle \cong \mathrm{A}_{4}$ then $\mathcal{X}_{5}$ is $\overline{\mathbb{F}}_{p}$-birationally equivalent to a curve $\mathcal{C}_{a}$ given in (10). If in addition $\mathcal{X}_{5}$ is defined over a finite field $\mathbb{F}_{q}$ with $q \equiv 1$ $(\bmod 4)$ and admits at least an $\mathbb{F}_{q}$-rational Weierstrass point $P=(t, 0)$, then $\mathcal{X}_{5}$ is $\mathbb{F}_{q}$-birationally equiavalent to $\mathcal{C}_{a}$.

Proof. Without loss of generality assume that $\operatorname{deg}(F(x))=12$. Lemma 4.1 shows that the roots of $F(X)$ form an orbit under the action of an automorphism group of $\overline{\mathbb{F}}_{p}(x)$ isomorphic to $A_{4}$, namely $\bar{H}=H /\left\langle\alpha_{1}\right\rangle$. Then there is an automorphism $\gamma$ of $\overline{\mathbb{F}}_{p}(x)$ such that

$$
\gamma \bar{H} \gamma^{-1}=\left\langle\theta_{1}, \theta_{2}\right\rangle \text {. }
$$

Hence $\gamma$ maps the roots of $F(X)$ to an orbit $\Omega$ under the action of $\left\langle\theta_{1}, \theta_{2}\right\rangle$. If $t$ is a point in $\Omega$, then $\Omega$ consists of

$$
\begin{gathered}
t, \theta_{1}(t)=-t, \theta_{2}(t)=\frac{t-i}{t+i}, \theta_{2}^{2}(t)=-i \frac{t+1}{t-1}, \theta_{1} \theta_{2}(t)=-\frac{t-i}{t+i}, \theta_{2} \theta_{1}(t)=\frac{t+i}{t-i}, \theta_{1} \theta_{2} \theta_{1}(t)=-\frac{t+i}{t-i} \\
\theta_{2}^{2} \theta_{1}(t)=-i \frac{t-1}{t+1}, \theta_{1} \theta_{2}^{2}(t)=i \frac{t+1}{t-1}, \theta_{1} \theta_{2}^{2} \theta_{1}(t)=i \frac{t-1}{t+1}, \theta_{2}^{2} \theta_{1} \theta_{2}(t)=-\frac{1}{t}, \theta_{1} \theta_{2}^{2} \theta_{1} \theta_{2}(t)=\frac{1}{t}
\end{gathered}
$$


Label these values with $z_{1}, \ldots, z_{12}$. Then $G(X):=\prod_{i=1}^{12}\left(X-z_{i}\right)$ coincides with

$$
\begin{array}{r}
X^{12}+\frac{-t^{12}+33 t^{8}+33 t^{4}-1}{t^{10}-2 t^{6}+t^{2}} x^{10}-33 X^{8}+2 \frac{t^{12}-33 t^{8}-33 t^{4}+1}{t^{10}-2 t^{6}+t^{2}} X^{6} \\
-33 X^{4}+\frac{-t^{12}+33 t^{8}+33 t^{4}-1}{t^{10}-2 t^{6}+t^{2}} X^{2}+1
\end{array}
$$

Since the roots of $F(X)$ are mapped to the roots of $G(X)$ by an automorphism of $\overline{\mathbb{F}}_{p}(x)$, the curve $\mathcal{X}_{5}$ is birationally equivalent to that of equation $Y^{2}=G(X)$, which clearly belongs to the family described in (10) for

$$
a=\frac{t^{12}-33 t^{8}-33 t^{4}+1}{t^{10}-2 t^{6}+t^{2}}=\frac{t^{12}-33 t^{8}-33 t^{4}+1}{t^{2}\left(t^{4}-1\right)^{2}} .
$$

Note that if $q \equiv 1(\bmod 4)$ then the group $H$ is defined over $\mathbb{F}_{q}$ and $\bar{H}$ is conjugated to $\left\langle\theta_{1}, \theta_{2}\right\rangle$ in $\operatorname{PGL}(2, q)$; see [46, Theorem A.8]. This implies that if the curve $\mathcal{X}_{5}$ admits a Weierstrass point $(t, 0)$ which is $\mathbb{F}_{q}$-rational then $\mathcal{X}_{5}$ is isomorphic over $\mathbb{F}_{q}$ to a curve of type $\mathcal{C}_{a}$ in (10).

\section{Other eXamples of MaXimal CURVES}

In this section we investigate other examples of maximal curves.

Definition 5.1. Let $p$ be an odd prime and $b \in \mathbb{F}_{p}$. We denote by $U_{p, b}$ the curve of the affine equation

$$
x^{6}+y^{6}+1+b x^{2} y^{2}=0 .
$$

Proposition 5.2. Let $p \geq 5$, then $U_{p, b}$ has genus 10 and $\operatorname{Jac}\left(U_{p, b}\right) \simeq_{\mathbb{F}_{p^{2}}} E_{1}^{3} \times E_{2} \times E_{3}^{3} \times E_{4}^{3}$, where

$$
\begin{aligned}
& E_{1}: y^{2}=x^{3}+3(b+6) x^{2}+3(b+3)(b+12) x+27(b+3)^{2}, \\
& E_{2}: y^{2}=x^{3}-3^{3} b^{2} x^{2}+2^{3} 3^{3} b\left(b^{3}+27\right) x-3^{3} 2^{4}\left(b^{3}+27\right)^{2}, \\
& E_{3}: y^{2}=x^{3}+2 b x^{2}+b^{2} x-2^{2} \\
& E_{4}: y^{2}=x^{3}-48 b^{2} x^{2}+768 b\left(b^{3}+27\right) x-4096\left(b^{3}+27\right)^{2} .
\end{aligned}
$$

Proof. By [51, Proposition 10], Jac $\left(U_{p, b}\right) \simeq_{\mathbb{F}_{p^{2}}} H_{1}^{3} \times H_{2} \times J a c\left(H_{3}\right)^{3}$, where

$$
\begin{array}{ll}
H_{1}: & y^{2}=((-b-3) x+3)(1-3 x(1-x)), \\
H_{2} & : x^{3}+y^{3}+1+b x y=0, \\
H_{3} & : \quad y^{2}=-\left(x^{3}+b x^{2}+4\right)\left(x^{3}+1\right) .
\end{array}
$$

By [16, Page 27], for $G_{a}: y^{2}=\left(x^{3}+a x^{2}-4\right)\left(-16 x^{3}+16\right), a \in \mathbb{F}_{p}$, we have that $\operatorname{Jac}\left(G_{a}\right) \simeq_{\mathbb{F}_{p^{2}}}$ $E_{3}^{\prime} \times E_{4}^{\prime}$, where

$$
\begin{aligned}
& E_{3}^{\prime}: y^{2}=x^{3}-a / 2+a^{2} / 16-1 / 16 \\
& E_{4}^{\prime}: y^{2}=16\left(a^{3}-27\right) x^{3}-48 a^{2} x^{2}+48 a x-16
\end{aligned}
$$


This shows that $\operatorname{Jac}\left(H_{3}\right) \simeq_{\mathbb{F}_{p^{2}}} E_{3} \times E_{4}$. Finally, it turns out that $H_{1}$ is birational to $E_{1}, H_{2}$ is birational to $E_{2}$. Then the claim follows.

Proposition 5.3. The curve $U_{p, b}$ in (25) is $\mathbb{F}_{p^{2}}$-maximal when

$$
(p, b) \in\{(89,58),(101,96),(131,100),(191,116),(227,69),(239,94),(251,3)\} .
$$

Proof. Direct computations done using the package MAGMA [9] for $p \leq 251$.

\section{ACKNOWLEDGMENTS}

The research of D. Bartoli and M. Giulietti was partially supported by the Italian National Group for Algebraic and Geometric Structures and their Applications (GNSAGA - INdAM). D. Bartoli was supported by Università degli Studi di Perugia - Fondo ricerca di base Esercizio 2015 - Project: Codici Correttori di Errori. M. Giulietti was supported by Università degli Studi di Perugia - Fondo ricerca di base Esercizio 2015 - Project: Geometrie di Galois, Curve Algebriche su campi finiti e loro Applicazioni. M. Kawakita was partially supported by JSPS Grant-in-Aid for Scientific Research (C) $17 \mathrm{~K} 05344$.

\section{REFERENCES}

[1] Anbar N., Bassa, A., Beelen, P.: A complete characterization of Galois subfields of the generalized GiuliettiKorchmáros function field, Finite Fields Appl., 48, 318-330 (2017).

[2] E. Alekseenk, S. Aleshnikov, N. Markin, A. Zaytsev, Optimal curves over finite fields with discriminant -19 , Finite Fields Appl. 17, 350-358 (2011).

[3] Arakelian, N., Tafazolian, S., Torres, F.: On the spectrum for the genera of maximal curves over small fields, Adv. Math. Commun. 12, 143-149 (2018).

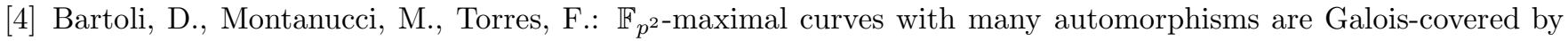
the Hermitian curve, submitted.

[5] Bartoli, D., Montanucci, M., Zini, G.: Multi point AG codes on the GK maximal curve, Des. Codes Cryptogr. 86, 161-177 (2018).

[6] Bartoli, D., Montanucci, M., Zini, G.: AG codes and AG quantum codes from the GGS curve, Des. Codes Cryptogr. 86, 2315-2344 (2018).

[7] Beelen, P., Montanucci, M.: A new family of maximal curves, Journal of the London Math. Soc. 98, 573-592 (2018).

[8] Beelen, P., Montanucci, M.: On subfields of the second generalization of the GK maximal function field, https://arxiv.org/abs/1811.00049.

[9] Bosma, W., Cannon, J., Playoust, C.: The Magma algebra system. I. The user language, J. Symbolic Comput. 24, 235-265 (1997).

[10] Bouw, I., Ho, W., Malmskog, B., Scheidler, R., Srinivasan, P., Vincent, C.: Zeta functions of a class of ArtinSchreier curves with many automorphisms, in: Directions in number theory, 87-124, Assoc. Women Math. Ser., 3, Springer, 2016.

[11] Brock, B.W.: Superspecial curves of genera two and three, Thesis (Ph.D.) Princeton University, (1993), 69 pp.

[12] Bröker, R., Lauter, K., Sutherland, A.V.: Modular polynomials via isogeny volcanoes. Mathematics of computation 81, 1201-1231 (2012).

[13] https://math.mit.edu/ drew/ClassicalModPolys.html (accessed 3 Jan 2019).

[14] Cossidente, A., Korchmáros, G., Torres, F.: Curves of large genus covered by the Hermitian curve, Comm. Algebra 28, 4707-4728 (2000). 
[15] Danisman, Y., Ozdemir, M.: On Subfields of GK and Generalized GK Function Fields. J. Korean Math. Soc. 52, 225-237 (2015).

[16] Djukanvic̀, M.: Split Jacobians and Lower Bounds on Heights, Thesis. https://openaccess.leidenuniv.nl/bitstream/handle/1887/54944/thesis.pdf?sequence=1

[17] Edge, W.L.: A canonical curve of genus 7, Proc. LMS(3) 17, 207-225 (1967).

[18] Edge, W. L.: Three plane sextics and their automorphisms, Canad. J. Math. 21, 1263-1278 (1969).

[19] Elkies, N.D.: The existence of infinitely many supersingular primes for every elliptic curve over $\mathbb{Q}$, Invent. Math. 89, 561-567 (1987).

[20] Elkies, N.D.: Shimura curve computations, in: "Algorithmic number theory" (Portland, OR, 1998, J. P. Buhler, ed.), Lecture Notes in Comput. Sci., 1423, Springer-Verlag, Berlin, 1998, 1-47.

[21] Fanali, S., Giulietti, M.: On maximal curves with Frobenius dimension 3, Des. Codes Cryptogr. 53(3), (2009) 165-174.

[22] Fanali, S., Giulietti, M.: Quotient curves of the GK curve, Adv. Geom. 12, 239-268 (2012).

[23] Fanali, S., Giulietti, M., Platoni, I.: On maximal curves over finite fields of small order, Adv. Math. Commun. 6, 107-120 (2012).

[24] Fricke, R.: Ueber eine einfache Gruppe von 504 Operationen, Math. Ann. 52, 321-339 (1899).

[25] Fuhrmann, R., Garcia, A., Torres, F.: On maximal curves, J. Number Theory 67, 29-51 (1997).

[26] Fuhrmann, R., Torres, F.: On Weierstrass points and optimal curves, Rend. Circ. Mat. Palermo Suppl. 51 (Recent Progress in Geometry, E. Ballico, G. Korchmáros Eds.), 25-46 (1998).

[27] Fuhrmann, R., Torres, F.: The genus of curves over finite fields with many rational points, Manuscripta Math. 89, 103-106 (1996).

[28] Garcia, A: Curves over finite fields attaining the Hasse-Weil upper bound, European Congress of Mathematics, Vol. II (Barcelona, 2000), Progr. Math. 202, Birkhäuser, Basel, 199-205 (2001).

[29] Garcia, A: On curves with many rational points over finite fields, Finite Fields with Applications to Coding Theory, Cryptography and Related Areas, Springer, Berlin, 152-163 (2002).

[30] Garcia, A., Güneri, C., Stichtenoth, H.: A generalization of the Giulietti-Korchmáros maximal curve, Advances in Geometry 10, 427-434 (2010).

[31] Garcia, A., Stichtenoth, H.: Algebraic function fields over finite fields with many rational places, IEEE Trans. Inform. Theory 41, 1548-1563 (1995).

[32] Garcia, A., Stichtenoth, H., Xing, C.P.: On subfields of the Hermitian function field, Compositio Math. 120, $137-170$ (2000).

[33] Garcia, A., Tafazolian, S.: Certain maximal curves and Cartier operators, Acta Arith. 135, 199-218 (2008).

[34] Giulietti, M., Hirschfeld, J.W.P., Korchmáros, G., Torres, F.: Curves covered by the Hermitian curve, Finite Fields Appl. 12, 539-564 (2006).

[35] Giulietti, M., Korchmáros, G.: A new family of maximal curves over a finite field, Math. Ann. 343, 229-245 (2009).

[36] Giulietti, M., Korchmáros, G.: Algebraic curves with many automorphisms, Advances in Math. 349, 162-211 (2019).

[37] Giulietti, M., Korchmáros, G.: Large p-groups of automorphisms of algebraic curves in characteristic $p$, J. Algebra 481, 215-249 (2017).

[38] Giulietti, M.; Montanucci, M.; Quoos, L.; Zini, G.: On some Galois covers of the Suzuki and Ree curves, J. Number Theory 189, 220-254 (2018).

[39] Giulietti, M., Quoos, L., Zini, G.: Maximal curves from subcovers of the GK-curve, J. Pure Appl. Algebra 220, 3372-3383 (2016).

[40] Gunby, B., Smith, A., Yuan, A.: Irreducible canonical representations in positive characteristic, Res. Number Theory 1, Art. 3, 25 pp, (2015).

[41] Kani, E., Rosen, M.: Idempotent relations and factors of Jacobians, Math. Ann. 284, 307-327 (1989). 
[42] Kazemifard, A., Tafazolian, S., Torres, F.: On maximal curves related to Chebyshev polynomials, Finite Fields Appl. 52, 200-213 (2018).

[43] Hansen, J.P.: Codes on the Klein quartic, ideals and decoding, IEEE Trans. Inf. Theory 33 923-925 (1987).

[44] Heegard, C., Little, J., Saints, K.: Systematic encoding via Gröbner bases for a class of algebraic-geometric Goppa codes, IEEE Trans. Inf. Theory 41, 1752-1761 (1995).

[45] Hidalgo, R.A.: Edmonds maps on the Fricke-Macbeath curve, Ars Math. Contemp. 8, 275-289 (2015).

[46] Hirschfeld, J.W.P., Korchmáros, G., Torres, F.: Algebraic Curves over a Finite Field. Princeton Series in Applied Mathematics, Princeton (2008).

[47] E. W. Howe, Curves of medium genus with many points, Finite Fields Appl. 47, 145-160 (2017).

[48] E. W. Howe, Quickly constructing curves of genus 4 with many points, in: Contemp. Math., 663, Amer. Math. Soc., 149-173, Providence, RI, (2016).

[49] Ihara, Y.: Some remarks on the number of rational points of algebraic curves over finite fields, J. Fac. Sci. Tokyo 28, 721-724 (1981).

[50] Joyner, D.: An error-correcting codes package, SIGSAM Commun. Comput. Algebra 39, 65-68 (2005).

[51] Kawakita, M.Q.: Wiman's and Edge's sextic attaining Serre's bound II, Algorithmic arithmetic, geometry, and coding theory, Contemp. Math. 637, 191-203 (2015).

[52] Kawakita M.Q.: Some Sextics of Genera Five and Seven Attaining the Serre Bound. In: Budaghyan L., RodrguezHenrquez F. (eds) Arithmetic of Finite Fields. WAIFI 2018. Lecture Notes in Computer Science, vol 11321, 264-271 (2018).

[53] Kawakita M.Q.: Certain Sextics with many rational points, Advances in Mathematics of Communications. 1, 289-292 (2017).

[54] Kawakita M.Q.: Wimans and Edges sextics attaining Serres bound, European Journal of Mathematics, 4, 330-334 (2018).

[55] Klein, F.: Über die Transformation siebenter Ordnung der elliptischen Functionen, Math. Ann. 14, 428-471 (1989).

[56] Kleiman, S.L.: Algebraic cycles and the Weil conjectures. In: "Dix esposés sur la cohomologie des schémas", pp. 359-386. North-Holland, Amsterdam, (1968).

[57] Korchmáros, G.; Torres, F.: On the genus of a maximal curve, Math. Ann. 323, 589-608 (2002).

[58] Lachaud, G.: Sommes d'Eisentein et nombre de points de certaines courbes algébriques sur les corps finis, C.R. Acad. Sci. Paris 30 5, 729-732 (1987).

[59] Ma, L., Xing, C.: On subfields of the Hermitian function fields involving the involution automorphism, Journal of Number Theory 198, 293-317 (2019).

[60] Macbeath, A.: On a curve of genus 7, Proc. Lond. Math. Soc. 15, 527-542 (1965).

[61] Matthews, G.L.: Codes from the Suzuki function field, IEEE Trans. Inf. Theory 50, 3298-3302 (2004).

[62] Matthews, G.L.: Weierstrass semigroups and codes from a quotient of the Hermitian curve, Des. Codes Cryptogr. 37, 473-492 (2005).

[63] Montanucci, M., Zini, G.: On the spectrum of genera of quotients of the Hermitian curve, Comm. Algebra 46, 4739-4776 (2018).

[64] Montanucci, M., Zini, G.: Some Ree and Suzuki curves are not Galois covered by the Hermitian curve, Finite Fields Appl. 48, 175-195 (2017).

[65] S. Nakajima, p-ranks and automorphism groups of algebraic curves, Trans. Amer. Math. Soc. 303, 595-607 (1987).

[66] Niitsuma, Y.: Counting Points of the Curve $y^{2}=x^{12}+a$ over a Finite Field, TOKYO J. MATH. VOL. 31(1), 59-94 (2008).

[67] Paulhus, J.: Elliptic factors in Jacobians of hyperelliptic curves with certain automorphism groups, in: ANTS X-Proceedings of the Tenth Algorithmic Number Theory Symposium, 487505, Open Book Ser. 1, Math. Sci. Publ., Berkeley, CA, (2013). 
[68] Rovi C., Izquierdo, M.: Maximal Curves Covered by the Hermitian Curve, https://www.manypoints.org/upload/1070601654.pdf.

[69] Rück, H.G., Stichtenoth, H.: A characterization of Hermitian function fields over finite fields, J. Reine. Angew. Math. 457, 185-188 (1994).

[70] Shaska, T.: Some special families of hyperelliptic curves, Journal of Algebra and its Applications, 3, 75-89 (2004).

[71] Skabelund, D.C.: New maximal curves as Ray class field over Deligne-Lusztig curves, Proc. Am. Math. Soc. 146, $525-540$ (2018).

[72] Southerland A.V.: On the evaluation of modular polynomials, preprint, arXiv: 1202.3985.

[73] Stichtenoth, H.: A note on Hermitian codes over $G F\left(q^{2}\right)$, IEEE Trans. Inf. Theory 34, 1345-1348 (1988).

[74] Stichtenoth, H.: Algebraic function fields and codes. In:Graduate Texts inMathematics, vol. 254. Springer, Berlin (2009).

[75] Stichtenoth, H., Xing, C.P.: The genus of maximal function fields, Manuscripta Math. 86, 217-224 (1995).

[76] Tate, J.: Endomorphisms of Abelian Varieties over Finite Fields, Inventiones Math 2, 134-144 (1966).

[77] Tafazolian, S., Teherán-Herrera, A., Torres, F.: Further examples of maximal curves which cannot be covered by the Hermitian curve, J. Pure Appl. Algebra 220, 1122-1132 (2016).

[78] Tafazolian, S., Torres, F.: A note on certain maximal curves, Comm. Algebra 45, 764-773 (2017).

[79] Tafazolian, S., Teherán-Herrera, A., Torres, F.: Further examples of maximal curves which cannot be covered by the Hermitian curve, J. Pure Appl. Algebra 220, 1122-1132 (2016).

[80] Tafazolian, S., Torres, F.: On the curve $y^{n}=x^{m}+x$ over finite fields, J. Number Theory 145, 51-66 (2014).

[81] Tafazolian, S., Torres, F.: On maximal curves of Fermat type, Adv. Geom. 13, 613-617, (2013).

[82] Tiersma, H.J.: Remarks on codes from Hermitian curves, IEEE Trans. Inf. Theory 33, 605-609 (1987).

[83] Top, J., Verschoor, C.: Counting points on the Fricke-Macbeath curve over finite fields, J. Th. des Nombres Bordeaux, to appear, (2016).

[84] Valentini, R.C., Madan, M.L.: A hauptsatz of L. E. Dickson and Artin-Schreier extensions, J. Reine Angew. Math. 318, 156-177 (1980).

[85] van der Geer, G.: Curves over finite fields and codes, European Congress of Mathematics, Vol. II (Barcelona, 2000), Progr. Math. 202, Birkhäuser, Basel, 225-238 (2001).

[86] van der Geer, G.: Coding theory and algebraic curves over finite fields: a survey and questions, Applications of Algebraic Geometry to Coding Theory, Physics and Computation, NATO Sci. Ser. II Math. Phys. Chem. 36, Kluwer, Dordrecht, 139-159 (2001).

[87] van Der Geer, G.: Counting curves over finite fields, Finite Fields Appl. 32, 207-232 (2015).

[88] van der Geer, G., Howe, E.W., Lauter, K.E., Ritzenthaler, C.: Tables of Curves with Many Points, (2009), http://www.manypoints.org, retrieved 10/03/2019.

[89] J. Wolfart, Triangle groups and Jacobians of CM type, preprint at http://www math.uni-frankfurt.de/ wolfart/Artikel/jac.pdf. (accessed 3 Jan 2019).

[90] Xing, C.P., Chen, H.: Improvements on parameters of one-point AG codes from Hermitian curves, IEEE Trans. Inf. Theory 48, 535-537 (2002).

[91] Xing, C.P., Ling S.: A class of linear codes with good parameters from algebraic curves, IEEE Trans. Inf. Theory 46, 1527-1532 (2000).

[92] Yang, K., Kumar, P.V.: On the true minimum distance of Hermitian codes. In: Coding Theory and Algebraic Geometry. Lecture Notes in Mathematics, vol. 1518, pp. 99-107. Springer, Berlin (1992). 
Dipartimento di Matematica e Informatica, Università degli Studi di Perugia, via Vanvitelli 1, 06123 Perugia, Italy

E-mail address: daniele.bartoli@unipg.it

Dipartimento di Matematica e Informatica, Università degli Studi di Perugia, via Vanvitelli 1, 06123 Perugia, Italy

E-mail address: massimo.giulietti@unipg.it

Division of Mathematics, Shiga University of Medical Science, Seta Tsukinowa-cho, Otsu City, SHIGA, 520-2192 JAPAN

E-mail address: kawakita@belle.shiga-med.ac.jp

Department of Applied Mathematics and Computer Science, Technical University of Denmark, Asmussens All, 2800 Kgs. Lyngby, Denmark

E-mail address: marimo@dtu.dk 